\title{
WestVirginiaUniversity
}

THE RESEARCH REPOSITORY @ WVU

Graduate Theses, Dissertations, and Problem Reports

2018

\section{Effects of disorder in models of active and inactive systems}

Joshua Thibault

Follow this and additional works at: https://researchrepository.wvu.edu/etd

\section{Recommended Citation}

Thibault, Joshua, "Effects of disorder in models of active and inactive systems" (2018). Graduate Theses, Dissertations, and Problem Reports. 7264.

https://researchrepository.wvu.edu/etd/7264

This Thesis is protected by copyright and/or related rights. It has been brought to you by the The Research Repository @ WVU with permission from the rights-holder(s). You are free to use this Thesis in any way that is permitted by the copyright and related rights legislation that applies to your use. For other uses you must obtain permission from the rights-holder(s) directly, unless additional rights are indicated by a Creative Commons license in the record and/ or on the work itself. This Thesis has been accepted for inclusion in WVU Graduate Theses, Dissertations, and Problem Reports collection by an authorized administrator of The Research Repository @ WVU. For more information, please contact researchrepository@mail.wvu.edu. 


\title{
Effects of disorder in models of active and inactive systems Joshua Thibault
}

\author{
Thesis submitted to the \\ Benjamin M. Statler College of Engineering and Mineral Resources \\ at West Virginia University
}

In partial fulfillment of the requirements for the degree of

\author{
Master of Science in \\ Mechanical Engineering
}

Stefanos Papanikolaou, Committee Chair, Ph.D.

Bruce Kang, Ph.D.

Terence Musho, Ph.D.

Department of Mechanical and Aerospace Engineering

Morgantown, West Virginia

2018

Keywords: Disordered Systems, Active Systems, Inactive Systems Microstructural Modelling of Disorder, Crack Formation, Crack Growth, Active Matter, Phase Transitions, Self-propelled particles

Copyright 2018 Joshua Thibault 


\title{
Abstract \\ Effects of disorder in models of active and inactive systems
}

\author{
Joshua Thibault
}

Disordered systems can be present in diverse contexts, such as material science, biology and social science. The statistical effects of disorder can vary according to whether the system is active or inactive. In general terms, the activity labels the capacity of local degrees of freedom to generate energy. The focus of this thesis is the demonstration of similarities and differences of disorder effects between active and inactive systems, by focusing on two basic examples.

Disorder in inactive systems is understood by focusing on the statistical behavior of a brittle heterogeneous alloy with disorder at the micrometer-scale. In this system, we study how microstructural disorder impacts crack formation and growth characteristics. The WeierstrassMandelbrot fractal function is used to generate a fluctuational variation in the critical strain energy release rate, a parameter used to determine damage in the phase field. An ellipsoidal notch is placed at the lateral edge of the sample and the sample is subjected to mode I loading via a defined strain rate. This is an investigational tool to study the dynamic transition from brittle, notch-based crack initiation to quasi-brittle, disorder-driven crack nucleation in the material bulk. This transition is identified for various disorder strengths in terms of the damage evolution and a discussion of the observations and analyses of this brittle to quasi-brittle transition in experiments is presented.

Disorder in active systems is explored by focusing on a basic model for bacteria populations, crowds of pedestrians or flocks of birds. A single drive force imparted on all the particles in a system can be approximated as what motivates the collective group's overall motion. The motion that accounts for the individualistic, random behavior of particles in a system is modeled by a runand-tumble, local velocity dependent, force. Run-and-tumble dynamics are considered a sufficient approximation of a particle's individual motion. For this system, a study is conducted in a channeltype flow with bi-directional traffic, analogous to humans in a tunnel-like space or airport terminal. A molecular dynamics model is used to describe the possible collective behaviors that these systems can form through self-organization from a quenched disordered orientation. A dynamic transition in the order of the particles within the simulation is studied. These simulations appear to have two competing effects: the individual motion of the particles due to the run-and-tumble effect and the overall drive force on the group of particles. The four identified phases of the disks as a function of increasing drive force are: a jammed state, a phase separated state, a mixed state, and a laning state. These transitions in phases are corroborated by visible drops in the average velocity of one type of particle and the six-fold disk ordering as a function of the drive force. 


\section{Acknowledgements}

I want to thank Dr. Papanikolaou for providing invaluable assistance on this project and providing me with a much-needed push throughout my academic career. I would like to thank him for allowing me to pursue a teaching assistant position with the college as it gives me great joy to interact with other students and help them achieve their academic goals.

I would like to acknowledge the use of the WVU High Performance Computing facilities for this project. Also, I would like to thank Dr. Papanikolaou for providing our lab with exceptional equipment that helped contribute to my success with this project.

Finally, I would like to thank my family and friends for being there for me. I don't know if I could have ever finished my master's degree without them. They helped drive and push me to achieve this magnificent feat. I am better for it and I thank them from the bottom of my heart for their love and support. 


\section{Table of Contents}

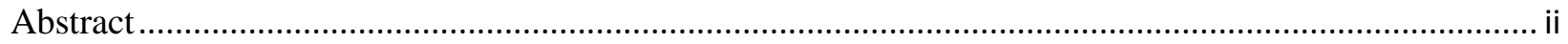

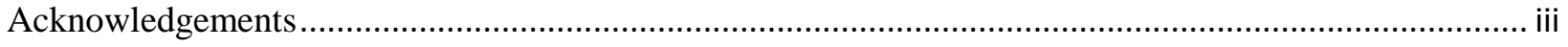

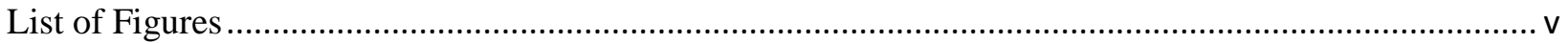

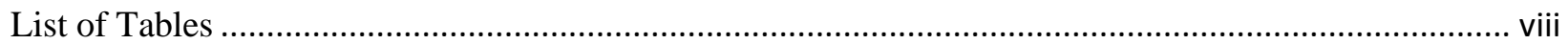

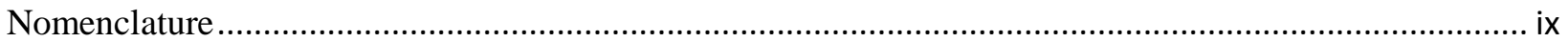

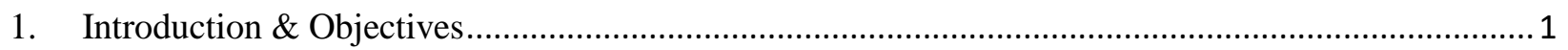

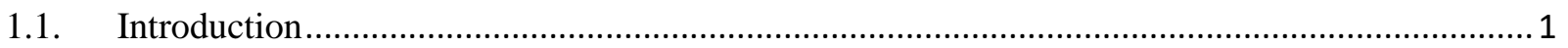

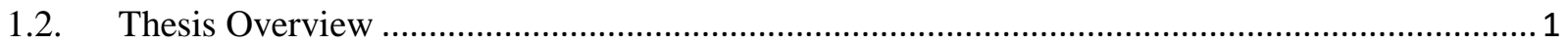

2. Inactive Disordered Systems: Fracture in disordered materials .................................................... 3

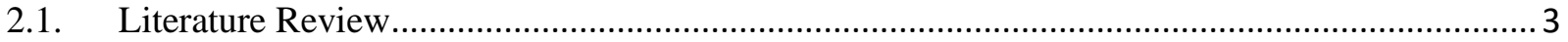

2.2. The Model: Surface Generation, DAMASK Material Point Model, and Phase Field Approach .8

i. 2D Disorder Generation for a Material Parameter...................................................................... 8

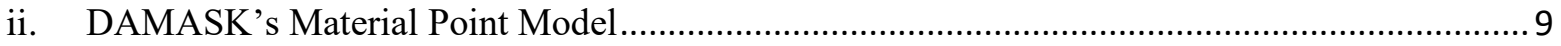

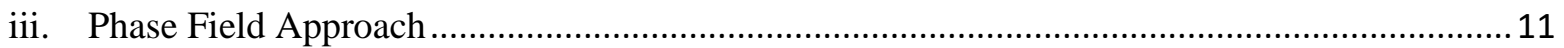

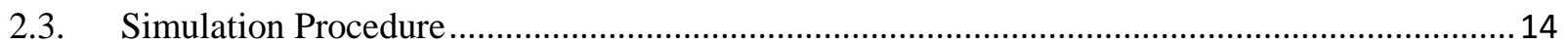

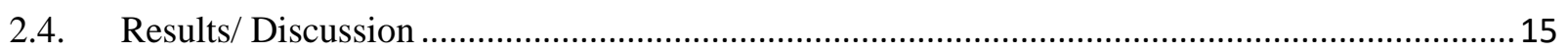

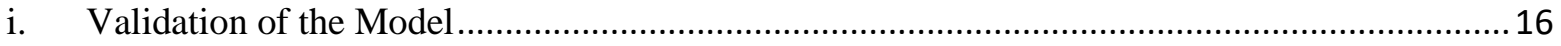

ii. Effect of Parameters that Control Material Disorder ............................................................17

iii. Width of Notch and its Effects on Crack Initiation and Propagation ......................................2 21

iv. Fracture Characteristics for a Fixed-Dimensioned Notch with Increasing Disorder.................23

v. Quantification of Disorder in terms of the Average and Maximum Stress ..............................27

3. Active Disordered Systems: Bidirectional flow of binary self-propelled particles...........................33

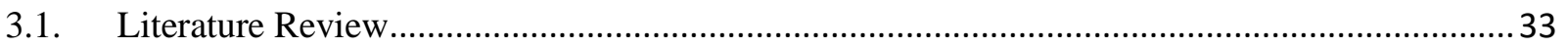

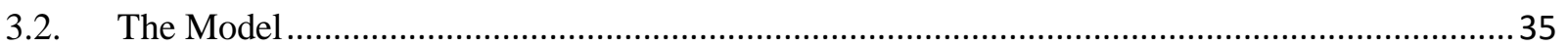

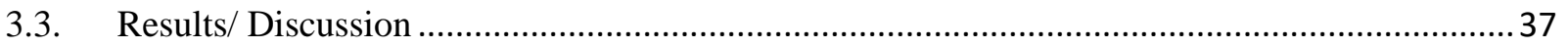

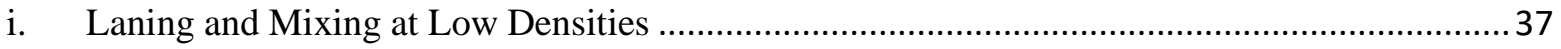

ii. Phases Observed at High System Densities............................................................................ 40

iii. Evolution of the Phase Diagram with Particle Density ....................................................... 47

4. Discussion: Disordered effects in active and inactive systems ….................................................51

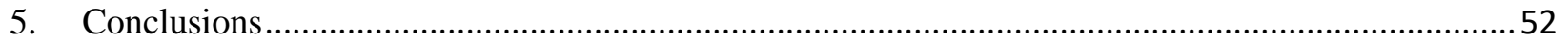

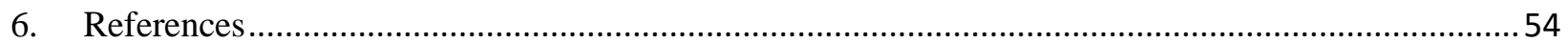




\section{List of Figures}

Figure 1: Microstructural length scales of alloys and superalloys: (a) and (b) are FEG-SEM surfaces (with visible crack) of High-Pressure Die-Cast Magnesium Alloys (El Kadiri et al. 2008). (c) and (d) are SEM images of a TiAl alloy where (c) is the crack and (d) is an image of the microstructure (Brotzu et al. 2014).

Figure 2:Microstructural length scales of alloys and superalloys (continued): (a) and (b) are magnified image of the microstructure of a Ni-based superalloy where (a) is the microstructure and (b) is a propagating crack along a grain boundary (Xie et al. 2011).... 5

Figure 3: Stochastic crack growth rates of various intermetallics: (a) Crack growth rates for various intermetallics and ceramic materials as a function of applied stress intensity factor from fig 5 in ref [Ritchie and Peters 2001]. (b) Crack growth rates of TiAl-based alloy for large and small cracks as a function of applied stress intensity factor from fig 4 in ref [Ritchie and Peters 2001].

Figure 4: Structure of the phase field approach and material point model: A schematic showing the basic steps for recursively solving the typical elasticity, plasticity and damage equations using fast and stable spectral solvers in the presence of disorder (Roters et al. 2012).

Figure 5: Basic dimensions with an induced disorder distribution: A disorder strength of $R_{\mathrm{G}}=0.2$ was chosen where the thickness of the sample, $\mathrm{L}_{x}$, is $8 \mu \mathrm{m}$, the width of the sample, $\mathrm{L}_{z}$, is $1.0 \mathrm{~mm}$, the length of the sample, $\mathrm{L}_{\mathrm{y}}$, is $0.5 \mathrm{~mm}, \mathrm{~W}$ is the initial notch width is $128 \mu \mathrm{m}, \mathrm{d}$ is $96 \mu \mathrm{m}$, and a is the constant major axis of $64 \mu \mathrm{m}$ (Papanikolaou et al. 2017, under review).

Figure 6: Satisfaction of convergence criterion: Simulations conducted at various system sizes with proportional notch widths to demonstrate resolution of the notch curvature and the occurrence of converging behavior during fracture (Papanikolaou et al. 2017, under review).....

Figure 7: Discretization approximation of the notch curvature: The sharp notch tip is present due to the discretization approximation of the notch's curvature where a single discrete unit is present. This highresolution curvature (2) is compared to a curvature at lower resolution (1) where this approximation dose not display the same characteristic (Papanikolaou et al. 2017, under review).

Figure 8: Effect of increasing notch width on crack nucleation for fractal dimension $D=2.85$ : Simulations of system size of $\mathrm{L}_{\mathrm{x}}=8 \mu \mathrm{m}, \mathrm{L}_{\mathrm{y}}=0.5 \mathrm{~mm}$, and $\mathrm{L}_{\mathrm{z}}=1.0 \mathrm{~mm}$ where $\mathrm{D}=2.85, \mathrm{R}_{\mathrm{G}}=0.8$, and (a) has a W of 16 $\mu \mathrm{m}$ (b) has a W of $128 \mu \mathrm{m}$ (c) has a $\mathrm{W}$ of $0.5 \mathrm{~mm}$ where the texture, damage and stress distributions are displayed, respectively (Papanikolaou et al. 2017, under review).

Figure 9: Disorder distribution for local fracture tendency and spatial profiles:: The progression of the disorder ratio, $\mathrm{R}_{\mathrm{G}}$, for $\mathrm{W}=0.125 \mathrm{~mm}$ with a system size of $\mathrm{L}_{\mathrm{x}}=8 \mu \mathrm{m}, \mathrm{L}_{\mathrm{y}}=0.5 \mathrm{~mm}$, and $\mathrm{L}_{\mathrm{z}}=1.0 \mathrm{~mm}$ where (a) has a disorder ratio of 0.1 , (b) has a disorder ratio of 0.2 , (c) has a disorder ratio of 0.4 , (d) has a disorder ratio of 0.8 , (e) and (f) are the probability density function (pdf) and cumulative distribution function (cdf), respectively, of the fluctuating phase field energy parameter of each node vs. the phase field energy parameter (Papanikolaou et al. 2017, under review).

Figure 10: Effect of notch width on crack initiation in a disordered crystal:: Simulations of system size of $\mathrm{L}_{\mathrm{x}}=8 \mu \mathrm{m}, \mathrm{L}_{\mathrm{y}}=0.5 \mathrm{~mm}$, and $\mathrm{L}_{\mathrm{z}}=1.0 \mathrm{~mm}$ where $\mathrm{D}=2.995, \mathrm{R}_{\mathrm{G}}=0.2$, and (a) has a W of $16 \mu \mathrm{m}$ (b) has a W of $64 \mu \mathrm{m}$ (c) has a W of $0.25 \mathrm{~mm}$ (d) has a W of $0.5 \mathrm{~mm}$ where the texture, stress and damage distributions are displayed (top, middle, bottom), respectively (Papanikolaou et al. 2017, under review)......

Figure 11: Effect of increasing disorder strength on crack nucleation: Simulations of system size of $\mathrm{L}_{x}=8$ $\mu \mathrm{m}, \mathrm{L}_{\mathrm{y}}=0.5 \mathrm{~mm}$, and $\mathrm{L}_{\mathrm{z}}=1.0 \mathrm{~mm}$ where $\mathrm{D}=2.995, \mathrm{~W}=0.125 \mathrm{~mm}$, and (a) has a $\mathrm{R}_{\mathrm{G}}$ of 0.05 (b) has a $\mathrm{R}_{\mathrm{G}}$ of 0.2 (c) has a $R_{\mathrm{G}}$ of 0.8 where the disorder, stress and damage distributions are displayed (top, middle, bottom respectively), respectively (Papanikolaou et al. 2017, under review).

Figure 12: Stress and damage progression in a disordered, notched, crystalline sample: Simulations of system size of $\mathrm{L}_{\mathrm{x}}=8 \mu \mathrm{m}, \mathrm{L}_{\mathrm{y}}=0.5 \mathrm{~mm}$, and $\mathrm{L}_{\mathrm{z}}=1.0 \mathrm{~mm}$ where $\mathrm{D}=2.995, \mathrm{~W}=0.125 \mathrm{~mm}$, and $\mathrm{R}_{\mathrm{G}}=0.8$ 
where (a) is the undamaged phase, (b) is the early damage phase, (c) is the late damage accumulation phase, (d) is the multiple crack nucleation event, and (e) is the crack propagation where the damage and stress distributions and are displayed (top, middle), respectively, and (f), (g), and (h) are the average stress (Pa) vs. strain, average damage vs. strain, maximum von Mises stress (Pa) vs. strain (bottom) respectively, where the five locations are identified on the curves (Papanikolaou et al. 2017, under review).

Figure 13: Effect of disorder on maximum stress: The maximum stress of samples of $L_{x}=8 \mu \mathrm{m}, \mathrm{L}_{\mathrm{y}}=0.5$ $\mathrm{mm}$, and $\mathrm{L}_{\mathrm{z}}=1.0 \mathrm{~mm}$ where $\mathrm{D}=2.995$ as a function of the average strain with respect to $\mathrm{R}_{\mathrm{G}}$ where (a) has a $\mathrm{R}_{\mathrm{G}}=0.0$, (b) has a $\mathrm{R}_{\mathrm{G}}=0.1$, (c) has a $\mathrm{R}_{\mathrm{G}}=0.2$, and (d) has a $\mathrm{R}_{\mathrm{G}}=0.4$ (Papanikolaou et al. 2017, under review).

Figure 14: Effect of disorder on average stress: The average stress of the specimens of $L_{x}=8 \mu \mathrm{m}, \mathrm{L}_{\mathrm{y}}=0.5$ $\mathrm{mm}$, and $\mathrm{L}_{\mathrm{z}}=1.0 \mathrm{~mm}$ where $\mathrm{D}=2.995$ as a function of the average strain with respect to $\mathrm{R}_{\mathrm{G}}$ where (a) has a $\mathrm{R}_{\mathrm{G}}=0.0$, (b) has a $\mathrm{R}_{\mathrm{G}}=0.1$, (c) has a $\mathrm{R}_{\mathrm{G}}=0.2$, and (d) has a $\mathrm{R}_{\mathrm{G}}=0.4$ (Papanikolaou et al. 2017, under review).

Figure 15: Quantification of the fluctuations at crack nucleation and growth of average stress: Simulations of sample size $L_{x}=8 \mu \mathrm{m}, L_{y}=0.5 \mathrm{~mm}$, and $L_{z}=1.0 \mathrm{~mm}$ where $\mathrm{D}=2.995$ and $\mathrm{W}=64 \mu \mathrm{m}$ as a function of the average strain where (a) is the average stress vs. average strain, (b) is the zoomed-in view of the fluctuations for disorder strengths 0.4 and 0.8 , and (c) is the difference in the rate of change of the average stress and the initial rate of change of linear increase in average stress vs. average strain (Papanikolaou et al. 2017, under review).

Figure 16: Significant Stress Events for Brittle and Quasi-brittle Fracture: The number and size of the stress events were measured as a function of $R_{G}$ where (a) is a series of histogram curves for various $W_{C}$ and (b) is a plot of the event size for various $W_{C}$ (Papanikolaou et al. 2017, under review). Note that the notches of widths: 16, 32, 128, and 256 microns only have one stress event for $R_{G} \leq 0.2$ while the notch width of 64 microns was observed to have two stress events for $R_{G}$ of 0.5 and 1.0. These events have a magnitude on the order of about $10^{5} \mathrm{~Pa}$. Also, for $\mathrm{R}_{\mathrm{G}}=0.4$, there are two stress events for all notch widths and both events share approximately the same event size for all notch widths.

Figure 17: Average velocity and corresponding slope and mobility at low density: (a) The average velocity per particle as a function of drift in a sample of particle density of 0.55 . The run-and-tumble particles have a run time of $1,10,100,1000$, and 10000. (b) The corresponding $d<V>/ d_{D}$ as a function of drive force where a peak in the slope moves to higher drive force as run time increases. (c) The mobility of the particles as a function of drive force (See also Reichhardt and Reichhardt 2015 and Fig 1 in Reichhardt et al. 2018, under review).

Figure 18: Mobility of various drive forces: The mobility, M, as a function of runtime for various drive forces for $\phi=0.55$ where (a) is a drive force of 2.0, (b) is a drive force of 4.0, (c) is a drive force of 6.0, and (d) is a drive force of 8.0 (See also Reichhardt and Reichhardt 2015 and Fig 3 in Reichhardt et al. 2018, under review).

Figure 19: Characteristic effect of drive force on the system: (a) shows the system's configuration at the beginning of the simulation. (b) shows the system's configuration during the velocity fluctuations. (c) shows the system's configuration after achieving a steady state behavior. (d) Various drive forces are plotted and the number of particles was defined as 1154, a system density of about $70 \%$, and the runtime was fixed at 20 (See also Carbone and Cozzoli 2010, Lattuada and Hatton 2011, Reichhardt et al. 2018, under review).

Figure 20: Characteristic effect of runtime on the system: The system's drive force and number of particles were held constant at 7.0 and 1154, respectively, and the runtime is varied. (a) defines the runtime as 50. (b) defines the runtime as 100. (c) defines the runtime as 500. (d) defines the runtime as 
1000. (e) defines the runtime as 5000. (f) defines the runtime as 10000 (See also Reichhardt and Reichhardt 2015, Bechinger et al. 2016, and Reichhardt et al. 2018, under review)

Figure 21: Possible Phases Observed in the System: (a) and (b) display the steady state phase of jamming particle configuration and the corresponding average velocity profile, respectively. (c) and (d) display the steady state phase separation of the particles and the corresponding average velocity profile, respectively (See also Reichhardt and Reichhardt 2016, Reichhardt and Reichhardt 2018, and Fig 12 in Reichhardt et al. 2018, under review).

Figure 22: Possible Phases Observed in the System (continued): (a) and (b) display the steady state continuously mixing configuration of the system and the corresponding average velocity profile, respectively. (c) and (d) display the steady state laning orientation of the system and the corresponding average velocity profile, respectively (See also Reichhardt and Reichhardt 2016, Reichhardt and Reichhardt 2018, and Fig 12 in Reichhardt et al. 2018, under review).

Figure 23: Average velocity and structure profiles of various run times: For one realization, a system of density $\varphi=70 \%$ with runtimes of $20,200,2000$, and 20000. (a) average velocity as a function of drive force and (b) structural measurement of the system at steady state as a function of drive force (See also Reichhardt and Reichhardt 2016, Reichhardt and Reichhardt 2018, and Fig 11 in Reichhardt et al. 2018, under review). Note that corresponding jumps in $\mathrm{P}_{6}$ structure and average velocity indicate the transition of a phase behavior.

Figure 24: Phase Diagram of the Active Matter System: The phase diagram uses 1154 particles in the simulations, about $\sim 70 \%$ and is for one realization.. I denotes the region in which laning behavior occurs. II denote the region in which mixing behavior occurs. III denotes the region in which phase separation occurs. And IV denotes the region in which jamming occurs (See also Reichhardt and Reichhardt 2016, Reichhardt and Reichhardt 2018, and Fig 18 in Reichhardt et al. 2018, under review). Note that the peak in figure 21 for $\tau_{\mathrm{r}}=20$ shows the phase change from IV (jammed state) at low $F_{d}$, to phase III (phase separated or large scale clustered) as $F_{d}$ is increased slightly. As drive force is continually increased, the activity of the individuals causes phase II (mixing) to occur. Finally, at large enough $F_{d}$ the systems enters an ordered, laning state where individual activity of the particles is mitigated by the overall global drive of the system. As $\tau_{\mathrm{r}}$ is increased, the mixing state becomes more prevalent at a wider range of $F_{d}$ for the system density of $70 \%$. 


\section{List of Tables}

Table 1: Phase parameters used in the simulations for the model material. ...........................................16 


\section{Nomenclature}

Inactive Disordered Systems

\begin{tabular}{|c|c|}
\hline$a$ & notch depth \\
\hline $\mathrm{C}_{11}, \mathrm{C}_{12}, \mathrm{C}_{44}$ & elastic constants \\
\hline $\mathbb{C}$ & elastic tensor \\
\hline$d$ & width of boundary condition - air layer \\
\hline$D$ & fractal dimension (W-M function) \\
\hline $\boldsymbol{F}$ & deformation gradient \\
\hline $\boldsymbol{F}_{e}$ & elastic deformation \\
\hline$F_{p}$ & plastic deformation \\
\hline$g^{\alpha}$ & slip resistance stress for a slip system $\alpha$ \\
\hline$g_{\infty}^{\alpha}$ & saturation stress \\
\hline$G$ & variance (W-M function)/ \\
\hline & strain energy release rate (DAMASK) \\
\hline$\delta G_{C}, G_{C}$ & critical strain energy release rate \\
\hline$\Delta G_{C}$ & local component of the critical strain energy release rate \\
\hline$h_{0}$ & hardening parameter \\
\hline$h_{\alpha \beta}$ & hardening matrix \\
\hline I & identity matrix \\
\hline$J_{I C}$ & critical J-integral value \\
\hline$K_{I C}$ & critical stress intensity factor \\
\hline$L$ & sample length (W-M function) \\
\hline$L_{p}$ & plastic velocity gradient \\
\hline$L_{x}$ & sample's thickness \\
\hline$L_{y}$ & sample's width \\
\hline$L_{z}$ & sample's length \\
\hline$m$ & ridge index (W-M function)/ \\
\hline & constant (convergence criteria)/ \\
\hline & order of the phase field potential (DAMASK) \\
\hline$M$ & superpose ridges parameter (W-M function) \\
\hline$n$ & frequency index (W-M function)/ \\
\hline & stress exponential (DAMASK) \\
\hline$n$ & unit vector along the slip plane normal \\
\hline $\boldsymbol{P}$ & first Piola-Kirchhoff stress \\
\hline$R_{G}$ & disorder strength ratio \\
\hline$s$ & unit vector along the slip direction \\
\hline$S$ & second Piola-Kirchhoff stress \\
\hline$w$ & hardening parameter \\
\hline$W$ & notch width \\
\hline$X$ & dissipation potential \\
\hline$\alpha$ & index of a slip system \\
\hline$\gamma$ & scaling parameter (W-M function) \\
\hline$\dot{\gamma}^{\alpha}$ & slip rate \\
\hline$\dot{\gamma}_{o}$ & reference shear rate \\
\hline$\varepsilon$ & strain \\
\hline
\end{tabular}




$\begin{array}{cl}\varepsilon_{e q} & \text { tolerance } \\ \sigma & \text { stress } \\ \tau^{\alpha} & \text { resolve shear for a slip system } \\ \phi & \text { random phase (W-M function)/ } \\ & \text { damage parameter (DAMASK) } \\ \phi_{l} & \text { local damage } \\ \psi & \text { free energy } \\ \psi_{D} & \text { damage free energy } \\ \psi_{E} & \text { elastic free energy } \\ \psi_{E} & \text { stored elastic energy density } \\ \psi_{P} & \text { plastic free energy }\end{array}$

Active Disordered Systems

$\begin{array}{cl}d & \text { distance between two radii } \\ \widehat{\boldsymbol{d}} & \text { displacement vector between two radii } \\ \boldsymbol{F}_{d}^{i} & \text { external drive force } \\ \boldsymbol{F}_{m}^{i} & \text { motor force } \\ \boldsymbol{F}_{p p}^{i} & \text { particle to particle interaction force } \\ k & \text { spring constant } \\ L & \text { system size dimension } \\ N & \text { number of disks/ particles } \\ N_{d} & \text { number of interacting particles } \\ N_{v} & \text { number of velocity data points } \\ P_{6} & \text { density for sixfold coordinated vortices } \\ R & \text { radius of disk } \\ S_{x} & \text { x dimension of system size } \\ S_{y} & \text { y dimension of system size } \\ \boldsymbol{v}_{i} & \text { resultant velocity of a particle } \\ V & \text { total velocity of one type of particles } \\ Z_{i} & \text { particle coordination number } \\ & \\ \eta & \text { drag coefficient } \\ \phi & \text { system density } \\ \tau_{r} & \text { runtime } \\ \Theta(x) & \text { Heaviside function }\end{array}$




\section{Introduction \& Objectives}

\subsection{Introduction}

Disordered systems can range from large crowd dynamics to fracture characteristics in alloyed metals. Disorder can have qualitatively different effects depending on the character of the complex system, which may be generally classified into two categories: active and inactive (Lifshits et al. 1988). Active matter systems are composed of self-propelled particles such as flocks of birds, schools of fish, bacteria and crowds of people (Moussaid et al. 2009 and Couzin and Franks 2003). This feature magnifies the importance of disorder in initial flow directions, allowing the system to continually maintain this disorder characteristic. However, due to the changing structure of an assembly of these motor-like driven particles, they may become ordered, leading to phase transitions in behavior (Reichhardt and Reichhardt 2018). These systems may be thought as having annealed disorder because some of the parameters in the system change randomly and evolve with time. For example, if these particles were to become jammed, then the system would be considered ordered. However, increasing the activity and tuning other parameters in the system, different phases may arise in the overall structure of the system. Opposite of active systems, inactive systems have a quenched, disordered state where their randomness is fixed within the system and with respect to time such as polysaccharides, glass and crystallized structures (Turnbull 1969). For example, any heterogeneous material can be considered disordered. During the alloying manufacturing process, the microstructure develops fluctuating material properties due the inability to maintain a homogeneous environment during mixing, forming, and cooling. As a result, the physical properties are affected throughout the metal including the predictability of failure characteristics (Pampillo 1975). Though both categories of disordered systems have been explored, there are several topics which remain of focus in disordered systems (Bunde and Havlin 2012).

\subsection{Thesis Overview}

This thesis covers two different types of systems: active and inactive. Chapter 2 focuses on inactive disordered systems. More specifically, this class of systems may be used in fracture of heterogeneous materials that exhibit disorder within the microstructure. We focus exclusively on the quantification of disorder and classification of fracture from a heterogeneous sample with an engineered notch. The notch curvature is a parameter that is varied and used as a tool to study the effects of microstructural variability on fracture. We use a model material similar to nickel 
aluminide ( $\mathrm{NiAl}$ ) that can be considered an alloy with plastically deforming characteristics at small scales. We begin by reviewing some related topics to this research (see Section 2.1). Next, the model (see Section 2.2) used to calculate the fracture behavior is explained via the 2D disorder generation for a material parameter (see Section 2.2.i), the material point model (see Section 2.2.ii), and the phase field approach (see Section 2.2.iii). We introduce the experimental protocol used to quantify disorder at various disorder strengths and notch widths (see Section 2.3). Then, we discuss some of the observations seen in the study and how fracture is classified: brittle or quasibrittle (see Section 2.4).

Chapter 3 focuses on active disordered systems. Typically, these systems are referred to as collections of self-propelled particles. They are useful to model autonomous agents which can be directly related to large group behaviors of biological organisms or crowds of people. We study and analyze large crowd sociological behaviors, using a bi-directional, channel-type system. The primary motivation of this research is to understand the various large-scale behaviors present in a crowd. We focus on the classification of possible behaviors exhibited by this system by sweeping the parameter space: drive force and run-and-tumble force. We begin by discussing the overall effects of disorder, like dynamic phase transitions and various structural behaviors (see Section 3.1). Next, this thesis explains the model, discussing the equations used to determine possible interactions between the individual particles and overall motion (see Section 3.2). Further, we discuss emerging phenomena that are noted in the results section (see Section 3.3). Finally, this thesis concludes with the most critical similarities and differences of active and inactive disordered systems (see Chapter 4 and 5). 


\section{Inactive Disordered Systems: Fracture in disordered materials}

\subsection{Literature Review}

This section reviews how short crack growth effects failure in disordered materials. An example class of materials we may focus on are intermetallics and alloys such as TiAl-based alloys and NiAl-based alloys. These are disordered, given that they have quenched disorder characteristics within the microstructure due to manufacturing processes. Manufacturing processes have the greatest effect on a material's predicted properties. For intermetallics, alloying processes are used to produce alloys and superalloys that far exceed material strength properties of conventional metals. These characteristics make them ideal for high temperature applications in the automotive, aeronautical, and power generation industries (Kim and Dimiduk 1991, Kim 1994, Stoloff et al. 2000, and Clemens and Mayer 2013). It is important to understand every aspect of how a material will perform from strength properties to fracture characteristics. Our focus of research is on the fracture properties and how such an alloy or superalloy may fail under specific loading conditions. Predicting the fracture characteristics of these types of materials has proven difficult because intermetallics display several characteristic length scales which govern fracture characteristics and material strength properties (Dimiduk 1991, Kim 1994, Dimiduk 1999, and Kim and Campbell et al. 1999).

These length scales are the cause of the chaotic crack growth rates which can span several orders of magnitude. Furthermore, this occurs on a length scale of a few microns in intermetallics shown in Figure 1 and 2. This characteristic is not exclusive to the particular material we intend to study. Instead, this characteristic is exhibited in all alloys. Figure 1 and 2 display several types of alloys and superalloys that verify this assertion. Figure 1 (a) and (b) are microstructural images of die-cast Magnesium $(\mathrm{Mg}$ ) alloys where disorder can be seen at a few microns. Figure $1 \mathrm{~b}$ exhibits hairline cracks that appear in the microstructure and propagate in a nonlinear manner, but follows inhomogeneities, grain-boundaries, and microcracks within the material. Figure $1 \mathrm{c}$ and $1 \mathrm{~d}$ and figure $2 \mathrm{a}$ and $2 \mathrm{~b}$ show titanium $(\mathrm{Ti})$ and nickel $(\mathrm{Ni})$-based superalloys which display analogous microstructural length scale characteristics. The disorder in these superalloys, also, is observed at the microscale where cracks propagate in a stochastic fashion, similar to figure $1 \mathrm{~b}$. Furthermore, they are subject to the same flaws such as inhomogeneities or grain boundaries during crack growth 
that are considered in Mg-alloys. However, the disorder length scale for $\mathrm{Ti}^{1}$-alloys can be controlled through grain size.

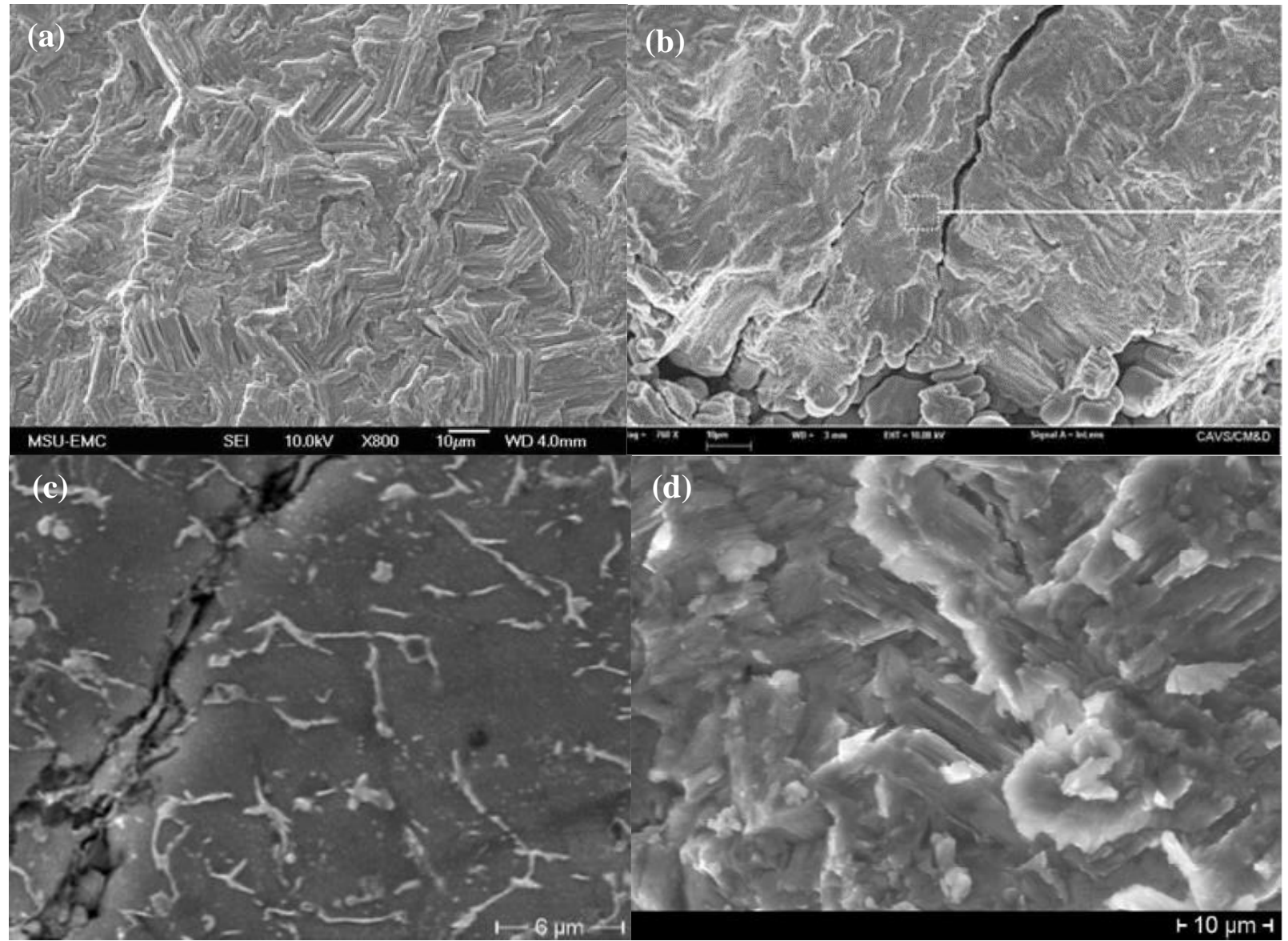

Figure 1: Microstructural length scales of alloys and superalloys: (a) and (b) are FEG-SEM surfaces (with visible crack) of High-Pressure Die-Cast Magnesium Alloys (El Kadiri et al. 2008). (c) and (d) are SEM images of a TiAl alloy where (c) is the crack and $(d)$ is an image of the microstructure (Brotzu et al. 2014).

\footnotetext{
${ }^{1}$ There are several TiAl-based alloys. The chemical composition can range from: Ti-(45-48\%) Al-(1-10\%) M- (at. $\%$ ) where $\mathrm{M}$ can be at least one of the following V, Cr, Mn, Nb, Ta, W, or Mo. Furthermore, these alloys can be divided into single phase $\gamma$-TiAl and two phase $\gamma-\mathrm{TiAl}+\alpha 2$-Ti3Al alloys. Depending on the alloy element and phases included, various material properties can be manipulated. For example, single phase $\gamma$ alloys contain $\mathrm{Nb}$ or Ta that promote strengthening and oxidation resistance. Also, various characteristics have been improved in two phase alloys such as increased ductility ( $\mathrm{V}, \mathrm{Cr}$, and $\mathrm{Mn}$ ), enhanced hot workability (B and $\mathrm{Mg}$ ), increased creep resistance ( $\mathrm{W}$ and $\mathrm{C}$ ), and enhanced room-temperature tensile elongation (reduced $\mathrm{O}$ ). Therefore, the application and material properties determine the alloying element used.
} 


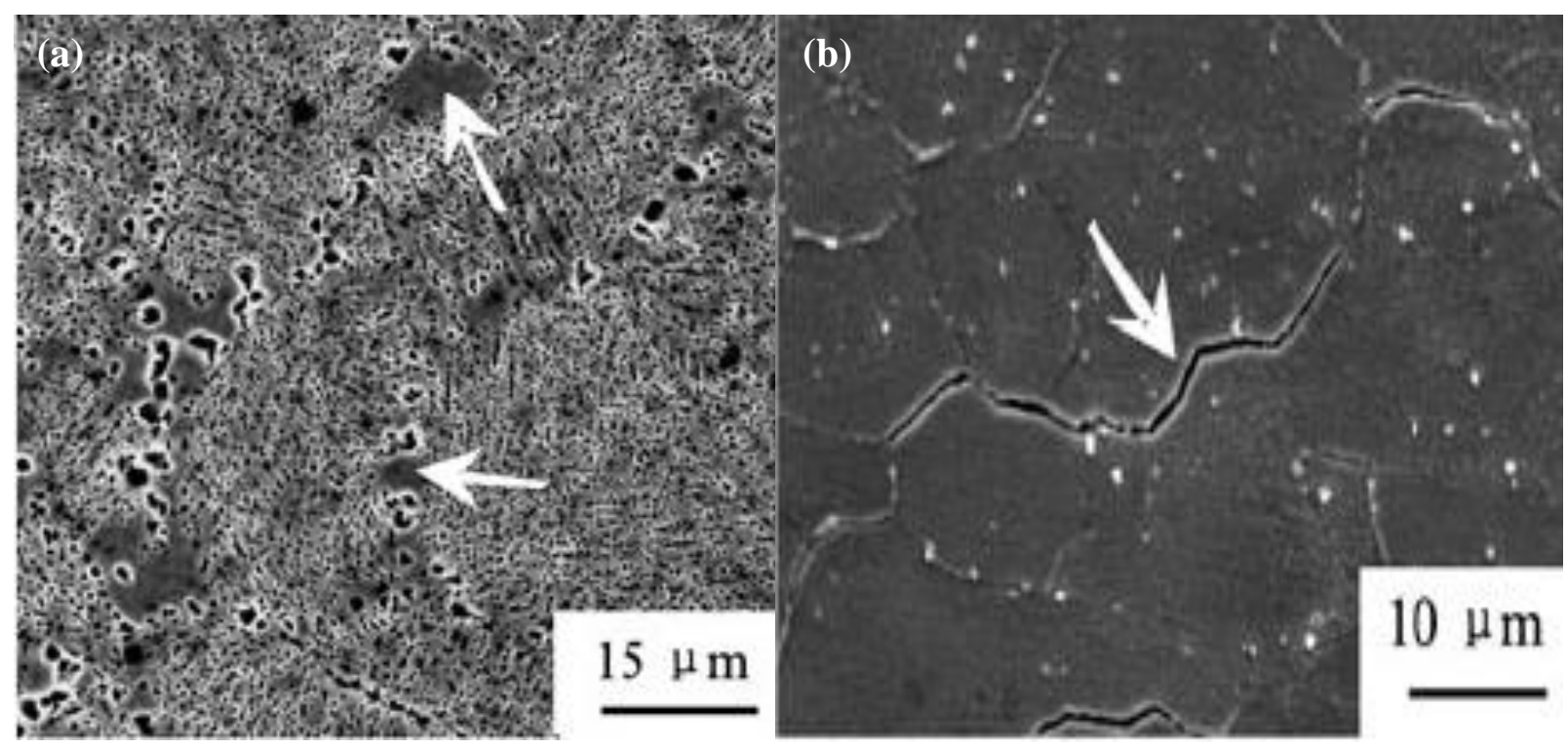

Figure 2:Microstructural length scales of alloys and superalloys (continued): $(a)$ and (b) are magnified image of the microstructure of a Ni-based superalloy where $(a)$ is the microstructure and $(b)$ is a propagating crack along a grain boundary (Xie et al. 2011).

For example, traditional casting techniques and alloying additives like $\mathrm{TiB}_{2}$ allow for grain sizes of about 800 microns in length (Hu 2001 and Han et al. 2015). While more modern techniques have improved such that grain sizes have decreased to about 2 microns (Lapin 2009 and Vajpai and Ameyama 2013) for fine-grained Ti-rich TiAl alloy compacts.

These alloying processes can determine the scale at which disorder is present in the microstructure. As a result, quenched disordered effects cause these alloys to display stochastic fracture characteristics, especially when cracks are short. 

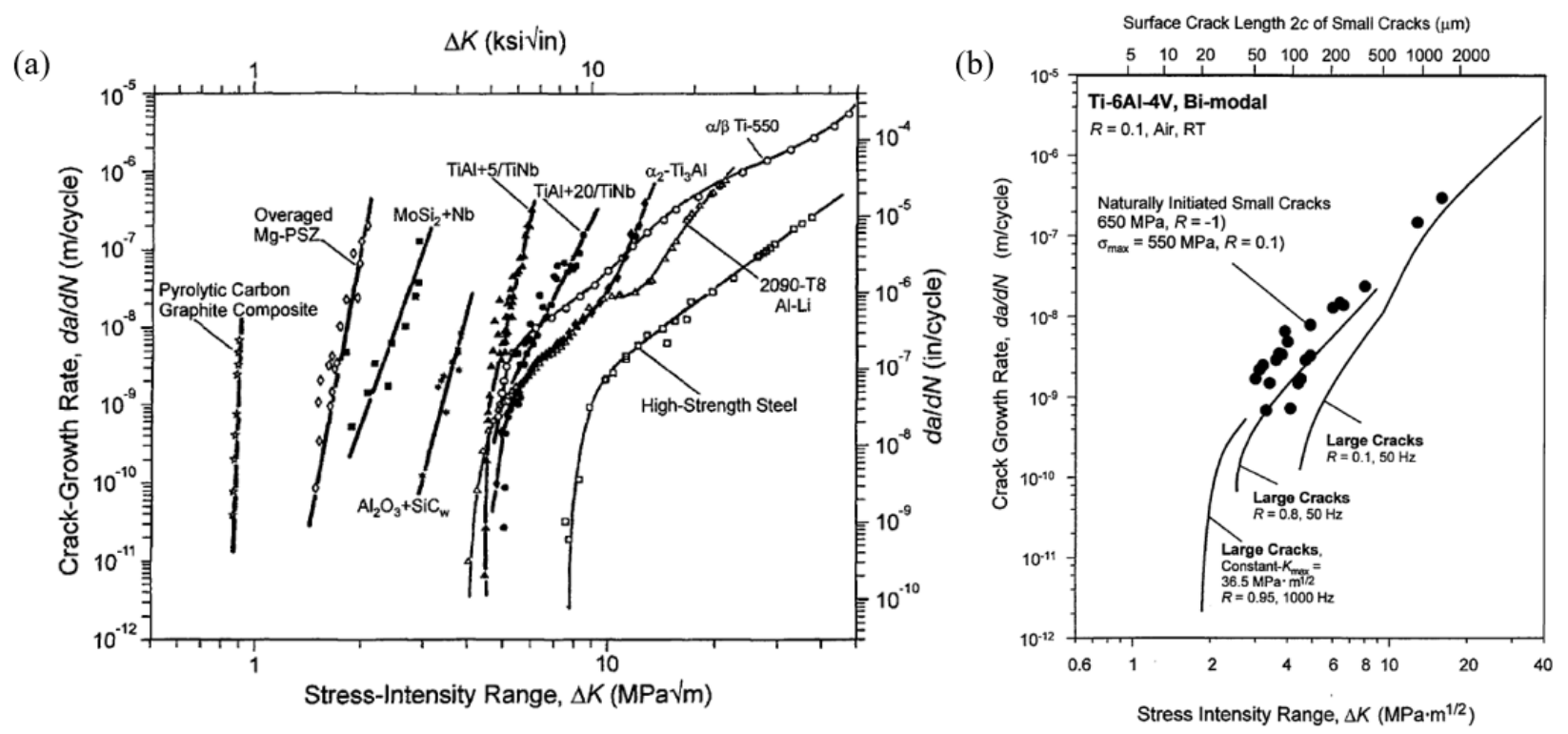

Figure 3: Stochastic crack growth rates of various intermetallics: (a) Crack growth rates for various intermetallics and ceramic materials as a function of applied stress intensity factor from fig 5 in ref [Ritchie and Peters 2001]. (b) Crack growth rates of TiAl-based alloy for large and small cracks as a function of applied stress intensity factor from fig 4 in ref [Ritchie and Peters 2001].

Consequently, short crack growth rates can extend several orders of magnitude (Figure 3) (approximately $10^{-11}$ to $10^{-6} \mathrm{~m} /$ cycle) (Ritchie and Peters 2001). Furthermore, such disorder characteristics are expected to evolve with fatigue loading. In this thesis, we research the possibility of quantifying and detecting disorder within the microstructure, using several free parameters to track damage evolution.

Microstructural heterogeneity is typically a result of alloying processes used to combine these intermetallics, and thereby, indispensable. On the macro scale, these alloys are regarded as brittle at room temperature. However, if we were to observe these alloys at the micron and submicron scale, then we would note the presence of crystal plasticity in the fracture characteristics. Therefore, it is necessary that any effort to model crack initiation and fracture should address this regime's behavior. These similar fracture characteristics to the plastic regime could be considered a result of microstructural disorder. So, considering disorder at the microscale, one can predict crack initiation and fracture characteristics. The principal question is: How can one quantify the effects of disorder on fracture and the evolution with material deformation and processing? It is the target of this thesis to fundamentally understand whether short crack growth events in alloys and superalloys can serve as consistent indicators of fracture. If this can be done, then developing a procedure to identify such indicators is important. Given the limited understanding of short crack growth, it is, also, important to develop an approach that can 
systematically capture this regime. We visualize an experimental procedure for identifying and controlling the onset of such events for short crack growth from a quenched, disordered system.

In order to develop this protocol, a model material was chosen. It is considered a simplification of an intermetallic alloy such as Nickel Aluminude (NiAl). NiAl is well known for its brittle fracture characteristics at large length scales and plastic, stochastic nature at a length scale of a few microns due to alloying processes (Lay and Yavari 1996, Herbold et al. 2011, and Wang et al. 2016). The disorder is modelled in a continuous manner where elastic and plastic deformation are considered at the micron and submicron scale. Therefore, it is natural to understand how these deformations compete at this scale.

The statistical nature of disorder has not been sufficiently explored as it relates to alloys and superalloys for short crack growth. During the formation of alloys, these processes produce several precipitates like $\gamma^{\prime}$ and $\gamma$ ' phases that effect local material properties (Tian et al. 2014). However, the disconnect comes from the lack of research into how this disorder can affect short crack growth during fracture. Due to the independence of linear elastic fracture mechanics (LEFM) (Zehnder 2012) on material length scales, it is possible to relate these characteristics to findings that have already been observed. A new class of fracture referred to as "Quasi-brittle" fracture does not obey typical brittle, elastic behaviors. Instead, these materials exhibit deviant fracture behavior that have plastically deforming characteristics. Observations have been noted for materials with large-scale disorder such as concrete (Walsh 1972). Many efforts have been made to characterize and predict this behavior (Bazant and Pfeiffer 1985, Schlangen and Van Mier 1992, Schlangen 1993, and Grote et al. 2001) and it was concluded that a nonlinear approach was needed for materials with a heterogeneous mesostructure. Furthermore, it is an objective of this thesis to contribute to the analysis and characterization of quasibrittle materials at these small scales.

The approach is based on a realistic way to classify the fracture behavior by developing an experimental protocol that produces a definable transition in fracture behavior. Due to advances in microfabrication techniques, namely focused ion beam (FIB), one can cut precise-dimensioned notches to the resolution length scale of sub-10nms (Tseng 2004 and Ochiai et al. 2010). By applying this technique in the protocol, one can design a repetitive experiment where notches of different curvature can be observed effecting the fracture behavior. While modifying the curvature of the notch tip, this thesis uses a fixed-depth notch to influence fracture when the notch is sharp enough. It is possible to characterize this transition as a function of the notch curvature. 


\subsection{The Model: Surface Generation, DAMASK Material Point Model, and Phase Field Approach}

The Düsseldorf Advanced Material Simulation Kit (DAMASK) is a versatile, open-source software which can be used to solve elastoplastic boundary value problems in order to simulate elasticity, plasticity, and damage evolution. This simulation software is very useful because of its ability to rationalize constitutive crystal plasticity equations in a continuum mechanical framework. This occurs by solving for the elastic and plastic deformation at each material point and homogenizing them into a cumulative deformation gradient. DAMASK uses a spectral method to solve for deformation with fast Fourier transforms (FFT). Fourier methods require a rectangulargridded mesh and periodic boundary conditions. Therefore, we apply a layer of air in contact with the notch surface, satisfying the periodic boundary conditions, in order to resolve equations for the damage evolution. DAMASK solves a material point model that converges to a solution at each timestep for various multiscale characteristics of the boundary value problem (Roters et al. 2012). In this section, we discuss: how disorder of the material is generated (Section 2.2.i), the structure of the material point model (Section 2.2.ii), and the phase field approach (Section 2.2.iii).

\section{i. 2D Disorder Generation for a Material Parameter}

The heterogeneity of the sample is considered as the induced disorder of material bulk. The Weierstrass-Mandelbrot (W-M) function allows for modeling of such macrostructural disorder using microscopic roughness in the critical strain energy release rate required which statistically quantifies the microstructural imperfections. More specifically, it creates a stochastic distribution of the fluctuating critical strain energy release rate (Weierstrass 1895 and Mandelbrot 1979).

$$
\begin{aligned}
& \Delta G_{c}(x, y, z)=L\left(\frac{G}{L}\right)^{D-3}\left(\frac{l n \gamma}{M_{1} M_{2}}\right)^{1 / 2} \sum_{m_{1}=1}^{M_{1}} \sum_{m_{2}=1}^{M_{2}} \sum_{n=0}^{n_{\max }} \gamma^{(D-4) n} \times\left\{\cos \left(\phi_{m_{1}, m_{2}, n}\right)-\cos \left[\frac{2 \pi \gamma^{n}\left(x^{2}+y^{2}+z^{2}\right)^{1 / 2}}{L} \times\right.\right. \\
& \left.\left.\left(\sin \left(\frac{y}{x}\right) \times \sin \left(\frac{\pi m_{1}}{M_{1}}\right) \times \cos \left(\left(\frac{z}{\left(x^{2}+y^{2}\right)^{1 / 2}}\right)-\frac{\pi m_{2}}{M_{2}}\right)+\cos \left(\frac{y}{x}\right) \times \cos \left(\frac{\pi m_{1}}{M_{1}}\right)\right)+\phi_{m_{1}, m_{2}, n}\right]\right\}
\end{aligned}
$$

Within the W-M function, Equation (1), the parameter, G, is defined as the variance of the spatial fluctuations, and the fractal dimension, D, exhibits a strong influence over the spatial fluctuations in the energy and stochastic characteristics. Therefore, the critical parameters that cause the most stochastic changes and effects the crack growth and damage evolution are G and D (Mandelbrot et al. 1984, Shanthraj et al. 2011, and Carney and Mecholsky 2013). However, there are other 
parameters that help manipulate the fracture behavior: $L$ is the sample length, $\gamma$ is a scaling parameter, $M_{1}$ and $M_{2}$ is the superposed ridges used to construct the surface for its roughness, $\phi_{m_{1}, m_{2}, n}$ is a random phase with $\mathrm{m}_{1}, \mathrm{~m}_{2}$, and $\mathrm{n}$ defining the ridge and frequency index, $\mathrm{n}$ is a frequency index that must control the max index considered, $\Delta G_{c}(x, y, z)$ is the fluctuational critical strain energy release rate, and $\mathrm{x}[\mathrm{m}]$ and $\mathrm{y}[\mathrm{m}]$ and $\mathrm{z}[\mathrm{m}]$ correspond to locations in the Fourier grid. The W-M function is used to manipulate the critical strain energy release rate, $G_{c}$ and provides a statistical distribution in order to emulate fracture behavior in disordered alloys.

\section{ii. DAMASK's Material Point Model}

DAMASK's material point model utilizes the typical constitutive elasticity modeling. Also, it models plasticity through the popular constitutive rate dependent, but also slip dependent, crystal plasticity formulation (Asaro and Lubarda 2006). The structure of the algorithm uses typical material point modeling.

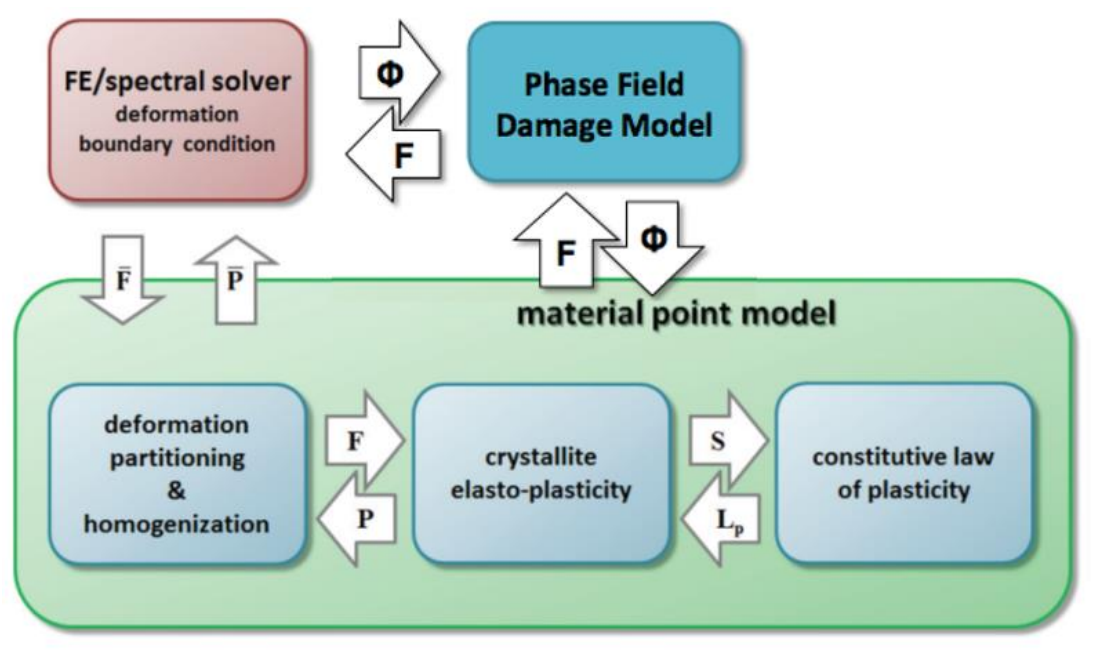

Figure 4: Structure of the phase field approach and material point model: A schematic showing the basic steps for recursively solving the typical elasticity, plasticity and damage equations using fast and stable spectral solvers in the presence of disorder (Roters et al. 2012).

The material point model rationalizes these elastic and plastic effects to determine the stresses at each point in the discretized specimen (Figure 4). Figure 4 presents a schematic of how this model determines deformation and damage evolution. The structure of the material point model begins by examining the deformation and the boundary conditions. At the initiation of the simulation, the deformation is considered to be zero. The boundary condition on the upper and lower edges are periodic in nature. However, due to the notched geometry on one of the lateral edges, the same approach cannot be applied for the sample. Therefore, we impose a layer of air at the notched edge. 
This boundary condition is considered periodic as both lateral edges have a layer of air to maintain the periodic boundary conditions necessary for FFT.

There are several levels to the solution procedure of the material point model. The spectral solver requires the average deformation gradient, $\overline{\boldsymbol{F}}$ and the corresponding average first PiolaKirchhoff stress, $\overline{\boldsymbol{P}}$, which has 6 variables due to its symmetry of the stress tensor. This information is necessary to determine the damage evolution in the phase field. Therefore, this model begins by partitioning the average deformation gradient into its individual deformation gradients, $\boldsymbol{F}(\boldsymbol{x})$, for each crystal in the aggregate. In DAMASK, the deformation gradient is a multiplicative decomposition of the elastic and plastic contributions: $\boldsymbol{F}(x)=\boldsymbol{F}_{e} \boldsymbol{F}_{p}$. The elastic deformation, $\boldsymbol{F}_{e}$, has nine variables and the plastic deformation, $\boldsymbol{F}_{p}$, has eight variables. At the crystallite level, the main objective is to determine the individual stresses, $\boldsymbol{P}$, at each material point. However, in order to solve for $\boldsymbol{P}$, the elastic and plastic effects must be accounted for. The second Piola-Kirchhoff stress, $\boldsymbol{S}$, implicitly drives the plastic velocity gradient, $\boldsymbol{L}_{\boldsymbol{p}}$, in order to solve the elasto-plasticity problem in the crystal (Roters et al. 2012). $\boldsymbol{S}$ has six variables because of its symmetry in the stress tensor. However, the plastic velocity gradient depends on the plasticity model used. The second Piola-Kirchhoff stress can be calculated as a function of the elastic deformation:

$$
S=\mathbb{C}\left(\boldsymbol{F}_{e}^{T} \boldsymbol{F}_{e}-\boldsymbol{I}\right) / 2
$$

Where $\mathbb{C}$ is the elasticity tensor and $\boldsymbol{I}$ is the identity matrix. We must also, rearrange the deformation gradient to yield the elastic deformation, $\boldsymbol{F}_{e}=\boldsymbol{F F}_{p}^{-1}$. The next step is to consider the constitutive level. The purpose of this level is to solve the constitutive law of plasticity. This begins by solving for the plastic velocity gradient which uses the second Piola-Kirchhoff stress. The slip rate, $\dot{\gamma}^{\alpha}$, is a function of $S$, and $\boldsymbol{L}_{p}$ is a function of this slip rate which has at least 12 variables and depends on lattice characteristics. To simplify the matrices, we account the active number of slip systems.

$$
\boldsymbol{L}_{p}=\sum_{\alpha} \dot{\gamma}^{\alpha} \boldsymbol{s}^{\alpha} \otimes \boldsymbol{n}^{\alpha}
$$

Where $\boldsymbol{s}$ and $\boldsymbol{n}$ are the unit vectors along the slip direction and slip plane normal, respectively, and $\alpha$ is the index of a slip system. We consider all 12 FCC slip systems which may become active in our single crystal with fixed crystalline orientation. However, the slip rate is guided by the basic phenomenological crystal plasticity constitutive equation. 


$$
\dot{\gamma}^{\alpha}=\dot{\gamma}_{o}\left|\frac{\tau^{\alpha}}{g^{\alpha}}\right|^{n} \operatorname{sgn}\left(\tau^{\alpha}\right)
$$

Where $\dot{\gamma}_{o}$ is the reference shear rate, $\tau^{\alpha}=\boldsymbol{S} \cdot\left(\boldsymbol{s}^{\alpha} \otimes \boldsymbol{n}^{\alpha}\right), n$ is the stress exponent and $g^{\alpha}$ is the slip resistance stress for a slip system $\alpha$. The hardening law is:

$$
\dot{g}^{a}=\sum_{\beta=1}^{12} \dot{\gamma}^{\beta} h_{0}\left|1-\frac{g^{\beta}}{g_{\infty}^{\alpha}}\right|^{w} \operatorname{sgn}\left(1-\frac{g^{\beta}}{g_{\infty}^{\alpha}}\right) h_{\alpha \beta}
$$

Where $w$ and $h_{0}$ are hardening parameters, $g_{\infty}^{\alpha}$ is the saturation stress, and $h_{\alpha \beta}$ is the hardening matrix which captures the interactions between different slip systems (Shanthraj et al. 2016). Once these parameters and equations are resolved and the plastic velocity gradient can be used to solve for the plastic deformation gradient: $\dot{\boldsymbol{F}}_{p}=\boldsymbol{L}_{p} \boldsymbol{F}_{p}$. The final step is to determine the first PiolaKirchhoff stress. We can relate $\boldsymbol{P}$ to the second Piola-Kirchhoff stress and the deformation gradient:

$$
\boldsymbol{P}=\boldsymbol{F S}
$$

The individual stresses, $\boldsymbol{P}$, are then homogenized into the average first Piola-Kirchhoff stress, $\overline{\boldsymbol{P}}$, used in the spectral solver. Perhaps the most important step is the homogenization of the stresses. While it is clear that the microstructure deformation cannot be expressed in this macrostructural model, the homogenized averages can be formulated in order to dictate the mechanical response of a representative volume element (RVE) (Roter et al. 2010). This process goes through several iterations until a convergence criterion is met for each incremental timestep. Because we use the Portable, Extensible Toolkit for Scientific Computation (PETSc) library, it is only necessary to achieve static equilibrium.

$$
\varepsilon_{e q} \geq \frac{\operatorname{RMS}(\operatorname{DIVP}(x))}{\|\overline{\boldsymbol{P}}\|} \times m
$$

Equation (7) is the convergence criterion where $\varepsilon_{e q}$ is the tolerance, $\overline{\boldsymbol{P}}$ is the average first PiolaKirchhoff stress, $m$ is a constant, and $\boldsymbol{P}(x)$ is the first Piola-Kirchhoff stress at each material point. This method is more convenient to implement in Fourier space by employing Parseval's theorem (Moulinec and Suquet 1998).

\section{iii. Phase Field Approach}

For the investigation of cracks, DAMASK utilizes a clear phase field approach (Aranson et al. 2000) where cracks are modeled by a phase with order parameter $\phi=0$ (fully damaged), while the material has $\phi=1$ (undamaged). $\phi$ couples proportionally to the elastic coefficients of the 
material, softening it as it is damaged. This approach utilizes a spectral method to resolve the elastic, plastic and damage equations during crack growth (Shanthraj et al. 2011 and Shanthraj et al. 2016). The material point model rationalizes the elastic and plastic effects in order to observe how damage evolves. The method for modelling damage in the phase field uses free energy equations to determine if certain criterion is met. The thermodynamically consistent free energy form is used.

$$
\psi=\psi_{E}+\psi_{D}+\psi_{P}
$$

Where $\psi_{E}, \psi_{D}$, and $\psi_{P}$ are the elastic, damage, and plastic free energy contributions, respectively. Elastic free energy is quite prevalent within the sample as it is loaded. The elastic energy is decomposed in a direct multiplicative manner to the simplest damage contribution $\left(\phi^{2}\right)$ and a pure distortion-dependent contribution:

$$
\psi_{E}=\phi^{2} \widetilde{\psi_{E}}\left(\nabla X, \boldsymbol{F}_{p}\right)
$$

At the undamaged state where damage hasn't occurred, the sole energy contribution to the sample is elastic free energy. In equation (9), it is a function of the stored elastic energy density, $\widetilde{\psi_{E}}$, and the phase field. The stored elastic energy density is dependent on the product of the dissipation potential, $X$, and plastic deformation. As the loading progresses, the strain energy release rate, $G$, reaches a critical point where the elastic free energy in the disordered distribution, locally, is converted to plastic free energy. The critical strain energy release rate, $G_{c}$, is how the model determines if permanent deformation has occurred. Locally, these areas will only exhibit damage and plastic free energy (Shanthraj et al. 2016).

The damage accumulation is determined by the Fourier grid variables as the critical strain energy release rate values are assigned. As the strain is increased, the mesh is used to track all respective energy contributions at each incrementally-increasing strain-step. With this progression, there is deformation in the sample that can be quantified. Damage is modelled using an isotropic brittle model based on rupture criterion where elastic strain energy, $G$, is the driving parameter for determining damage, $\phi: \phi_{l}=G_{c} / G . \phi_{l}$ is local damage. The damage free energy is nonzero only after the crack initiation event,

$$
\psi_{D}=1 / 2 G_{c} l|\nabla \phi|^{2}+G_{c} / l(1-\phi)^{m}+I_{[0,1]}(\phi)
$$

Where $G_{C}$ and $l$ are material constants and $m$ is the order of the phase field potential. Equation (10) represents how the damage free energy is determined. $G_{c}$ is the critical strain energy release rate, 
analogous to the work of fracture. In our phase field model, this amounts to the free energy contribution necessary for crack nucleation to occur, and $l$ is the resolution length scale which should correspond to a representative volume element (RVE) of the material (Shanthraj et al. 2016). In our simulations, we consider a fixed resolution length equal to 4 mesh units, which corresponds to $\sim 16 \mu \mathrm{m}$, given our considered sample dimensions. As damage evolves, there is a permanent deformation that, also, contributes to plastic free energy.

An analysis of the plastic free energy is quite nontrivial. Physically, it only occurs at the crack initiation and propagation areas. Furthermore, these are places at which permanent deformation occurs. Quantifying the plastic free energy contribution requires an indirect approach. We must first solve for the total free energy.

$$
\partial \boldsymbol{F}_{p} \psi=-\phi^{2} \boldsymbol{S} \boldsymbol{F}^{-T}
$$

However, we must reduce and simplify this equation into the familiar slip system based conjugate pair.

$$
-\partial \boldsymbol{F}_{p} \psi \cdot \dot{\boldsymbol{F}}_{p}=\sum_{\alpha} \partial \gamma^{\alpha} \psi \dot{\gamma}^{\alpha}, \text { where } \partial \gamma^{\alpha} \psi=-\phi^{2} \boldsymbol{S} \cdot\left(\boldsymbol{s}^{\alpha} \otimes \boldsymbol{n}^{\alpha}\right)=-\tau^{\alpha}
$$

As it can be seen, the plastic free energy is a function of several important parameters. It is necessary to consider the constitutive plasticity equation in order to solve for these parameters. Once the total free energy is solved for, we can rearrange equation (8) to find the plastic free energy of the sample. While it is useful to consider all contributions of free energy to the phase field, this model mainly focuses on whether criterion is sufficiently satisfied to assign damage to units on the Fourier grid.

In this work, we identify disorder in terms of the variability for the critical strain energy release rate $G_{c}$. We assume throughout that $G_{c}$ displays local, quenched and continuous fluctuations, as it would naturally happen in a multi-phased disordered metal alloy. Namely, we assume that $\delta G_{c} \rightarrow$ $\left\langle G_{c}\right\rangle+\Delta G_{c}(y, z)$ and the local fluctuations $\Delta G_{c}(y, z)$ are given in space through the W-M function, Equation (1), at a given fractal dimension $D$. The critical experimentally relevant parameter in these simulations is the degree of relative disorder fluctuations or the width of the distribution of $G_{c}$ with respect to the average critical strain energy release rate. If we define the standard deviation of the distribution as $\delta G_{c}$, then the important quantity to investigate would be $R_{G}=\delta G_{c} /\left\langle G_{c}\right\rangle$. In the next section we discuss the protocol developed in order to study the transition from brittle to quasibrittle fracture in a disordered alloy. 


\subsection{Simulation Procedure}

We simulate a transition from notch-driven crack initiation, characteristic of LEFM, to bulk crack nucleation, characteristic of quasibrittle fracture, using a model material that resembles a brittle material with plastically deforming properties. We use an integrated spectral phase field approach coupled constitutively to elastoplasticity through the DAMASK software (Roters et al. 2012). We modify it to introduce a microscopic roughness for the energy required for local damage. Elasticity is solved through a spectral solver which is fully parallelizable. Crystal plasticity is modeled through typical constitutive laws that take into account grain orientation, crystalline structure, and possible slip systems (Asaro 1983). Through the application of this multiscale, micromechanical analysis, the microstructure can be linked to the macroscopic material toughness energy.

In this thesis, we perform extensive simulations of a notched samples in a system of dimensions: $\mathrm{L}_{\mathrm{x}} \times \mathrm{L}_{\mathrm{y}} \times \mathrm{L}_{\mathrm{z}}$. The test samples have an assumed length scale of $1 \mathrm{~mm}$ per 256 units (essentially, each unit corresponds to $\sim 4 \mu \mathrm{m}$ ). $\mathrm{L}_{\mathrm{x}}$ and $\mathrm{L}_{\mathrm{z}}$ are held constant at $8 \mu \mathrm{m}$ and $1.0 \mathrm{~mm}$, respectively. $\mathrm{L}_{\mathrm{y}}$ is varied from $0.25 \mathrm{~mm}$ to $1.0 \mathrm{~mm}$. The ellipsoidal notch is placed along the $\mathrm{y}-$ axis at the edge of the specimen where the major axis, $\mathrm{a}=\mathrm{L}_{\mathrm{y}} / 8$, is held constant. However, in order to observe the transition from notch-driven crack initiation to bulk-induced crack nucleation, the notch width, $\mathrm{W}$, is varied at values from $16 \mu \mathrm{m}$ to $0.5 \mathrm{~mm}$, increasing the radius of curvature. In order to modify disorder, we consider quenched stochastic contribution in the fracture energy that controls the rate of local damage. Defining the ratio of the variance of local fracture energy with respect to the average fracture energy as $R_{G}$, we consider various cases where $R_{G}$ varies in the interval of $[0.0,0.8]$. The stochastic variable is picked through the Weierstrass-Mandelbrot function (Shanthraj et al. 2011) in which the fractal dimension, D, is either 2.85 or 2.995. As shown

in Figure 5, the sample has an induced disorder distribution that varies a parameter which we only consider in the damage evolution, according to the parameters and dimensions mentioned previously. In the next section, we discuss how this phase field model in a realistic elastoplastic environment can cause crack initiation and fracture at various notch widths and disorder strengths, keeping $\left\langle G_{c}\right\rangle$ fixed but changing the disorder ratio $R_{G}$. 


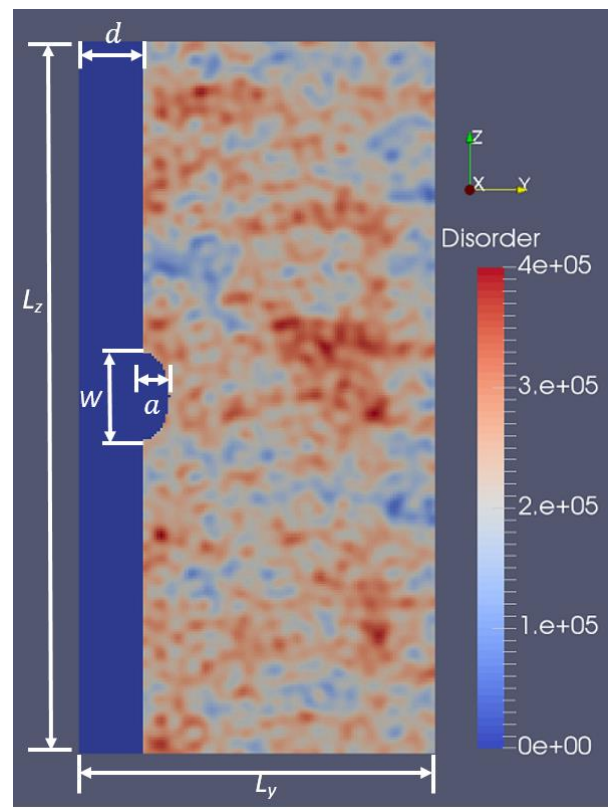

Figure 5: Basic dimensions with an induced disorder distribution: A disorder strength of $R_{G}=0.2$ was chosen where the thickness of the sample, $L_{x}$, is $8 \mu \mathrm{m}$, the width of the sample, $L_{z}$, is $1.0 \mathrm{~mm}$, the length of the sample, $L_{y}$, is $0.5 \mathrm{~mm}$, W is the initial notch width is $128 \mu \mathrm{m}$, $d$ is $96 \mu \mathrm{m}$, and a is the constant major axis of $64 \mu \mathrm{m}$ (Papanikolaou et al. 2017, under review).

\subsection{Results/ Discussion}

The intent of running these simulations is to identify the characteristic effect that the disorder has on the crack formation and growth. We do this by analyzing the stochastic damage and stress distributions in samples of various notch widths and disorder strengths, referring to the width of the distribution for the possible values of critical strain energy release rate. A crack initiates at the notch due to primarily elastic deformation until fracture and can be characterized as brittle. However, in the context of this experiment, when crack nucleation occurs in the bulk of the material, we believe this is due to mainly plastic effects, classifying the fracture as quasibrittle. The material parameters that govern this behavior are shown in Table 1. 
Table 1: Phase parameters used in the simulations for the model material.

\begin{tabular}{ccc}
\hline Property & Value (Model Material) & Units \\
\hline $\mathrm{C}_{11}$ & 106.75 & $\mathrm{GP} 2$ \\
$\mathrm{C}_{12}$ & 60.41 & $\mathrm{GPa}$ \\
$\mathrm{C}_{44}$ & 28.34 & $\mathrm{GPa}$ \\
$\dot{\gamma}_{0}$ & $1.0 \times 10^{-3}$ & $\mathrm{~s}^{-1}$ \\
$n$ & 20 & \\
$g_{0}$ & 31 & $\mathrm{MPa}$ \\
$g_{\infty}$ & 63 & $\mathrm{MPa}$ \\
$a$ & 2.25 & \\
$h_{0}$ & 75 & $\mathrm{MPa}$ \\
$h_{a \beta}$ & 0 &
\end{tabular}

Where $\mathrm{C}_{11}, \mathrm{C}_{12}$, and $\mathrm{C}_{44}$ are elastic constants and the remaining parameters are used in the plasticity model of DAMASK. These parameters are used in the constitutive elastic and plastic equations in order to determine the deformation and damage evolution of the sample.

\section{i. Validation of the Model}

A series of simulations were made in order to prove that we could achieve a condition referred to as grid independence. This goal would satisfy two criteria: It would confirm that the simulations we were running were at a sufficient size, eliminating any resolution issue in the disorder distribution as a result of the gridded mesh. Moreover, it would prove that DAMASK would produce accurate results, assuming the simulations achieved a convergent behavior based on the defined model material properties. In the context of this study, the system size is the sample's dimensions: $L_{x} x L_{y} x L_{z}$. As the sample size increases, the quality of the disorder distribution, also referred to as disorder's resolution, does the same. We began with a system of size 0.8 microns by 64 microns by 128 microns and doubled $L_{y}$ and $L_{z}$ until we observed convergence. 


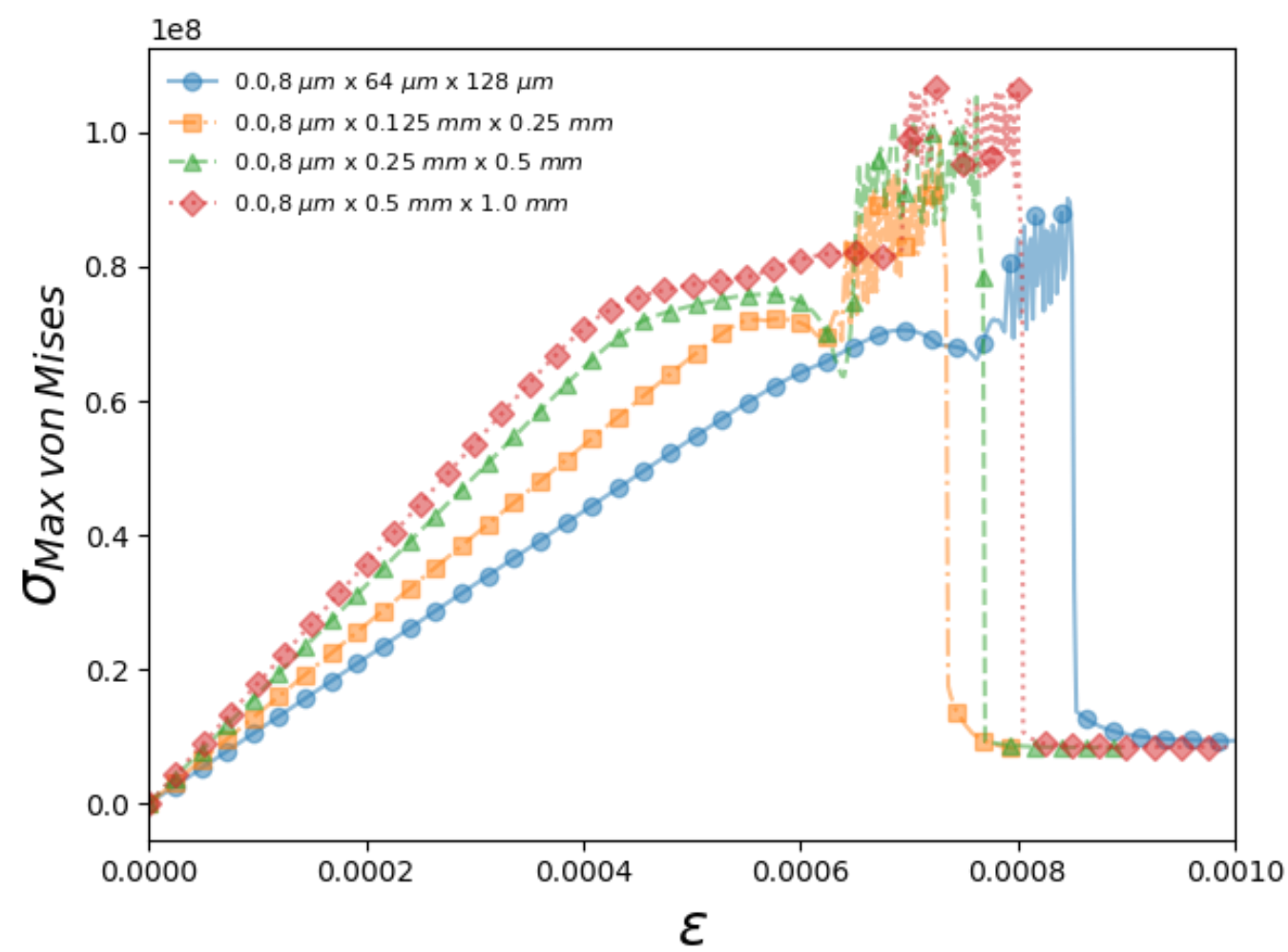

Figure 6: Satisfaction of convergence criterion: Simulations conducted at various system sizes with proportional notch widths to demonstrate resolution of the notch curvature and the occurrence of converging behavior during fracture (Papanikolaou et al. 2017, under review).

Figure 6 shows that convergence occurs at a minimum simulation size of $8 \mu \mathrm{m} \times 0.5 \mathrm{~mm} \times 1.0$ $\mathrm{mm}$. As a result, this observation dictated the system size used in this thesis, proving the system exhibits sufficient resolution to accurately model fracture.

\section{ii. Effect of Parameters that Control Material Disorder}

In the initial simulations, the fractal dimension, $\mathrm{D}$, is adjusted to naturally compete with the discretization unit at the notch tip as a result of the modelled notch tip curvature. 
Figure 7: Discretization approximation of the notch curvature: The sharp notch tip is present due to the discretization approximation of the notch's curvature where a single discrete unit is present. This high-resolution curvature (2) is compared to a curvature at lower resolution (1) where this approximation dose not display the same characteristic (Papanikolaou et al. 2017, under review).

The fractal dimension, D, is a parameter in the W-M function, Equation (1). Increasing this parameter increases the roughness of the fluctuations in the disorder distribution, giving locally more extreme values for the critical strain energy release rate. Effectively, this parameter increases the stochasticity of the critical strain energy release rate. The notch curvature is modelled in the simulations where a fixed-depth notch may exhibit a single discretized unit at the tip (Figure 7, curvature 2) of the notch for a system of size $8 \mu \mathrm{m} \times 0.5 \mathrm{~mm} \times 1.0 \mathrm{~mm}$. A lower resolution specimen is overlaid to show the different approximation of the notch curvatures. It doesn't exhibit the same tip approximation compared to the high-resolution system. However, convergence isn't achieved until the larger system size is reached. Therefore, increasing the fractal dimension was necessary to allow for the natural competition of disorder and the discretization of the notch.

Simulations were made at several fractal dimensions in order to determine its characteristic effect on the critical strain energy release rate values and the crack formation and growth features. 


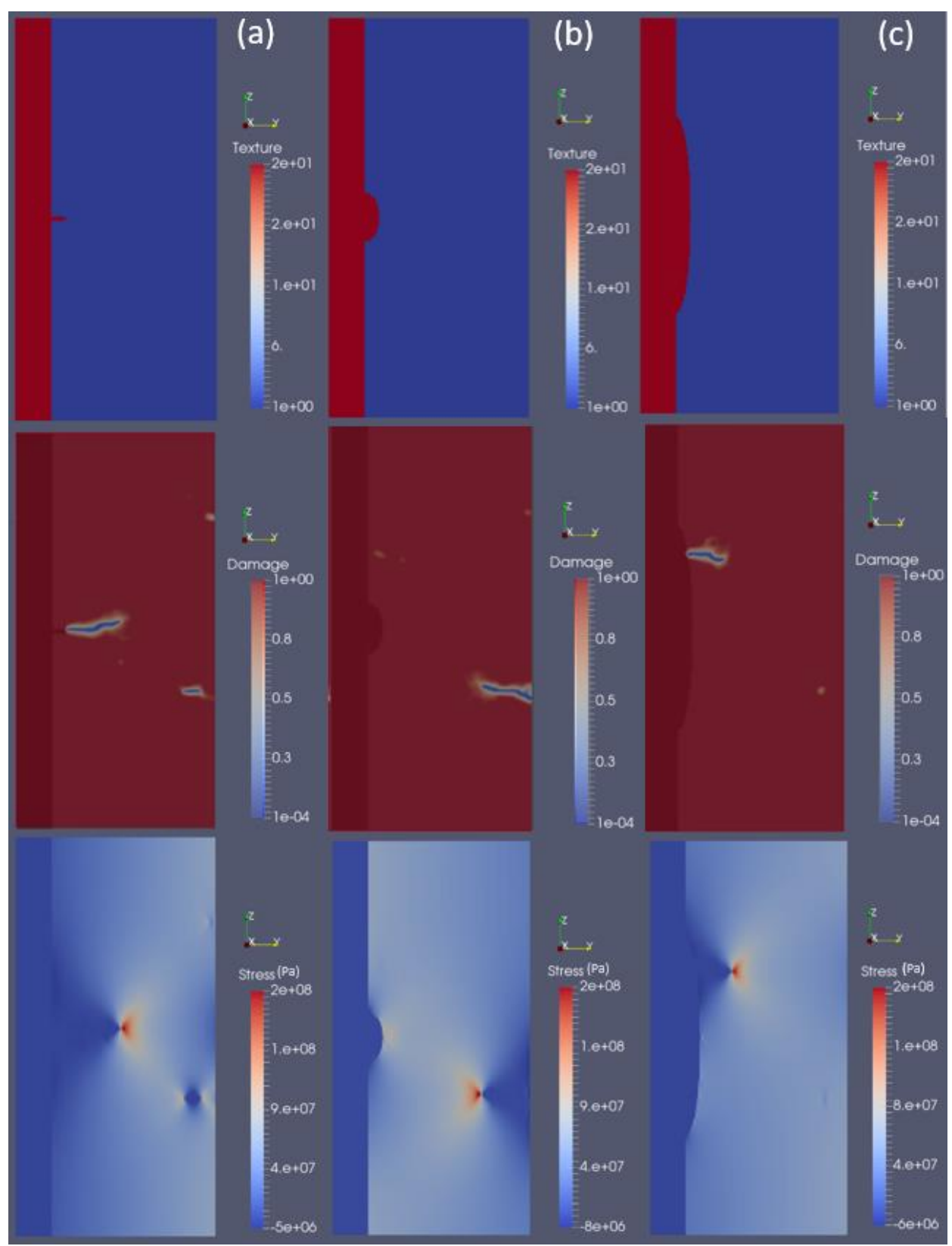

Figure 8: Effect of increasing notch width on crack nucleation for fractal dimension $\mathbf{D}=2.85$ : Simulations of system size of $L_{x}=8 \mu \mathrm{m}, L_{y}=0.5 \mathrm{~mm}$, and $L_{z}=1.0 \mathrm{~mm}$ where $D=2.85, R_{G}=0.8$, and (a) has a W of $16 \mu \mathrm{m}$ (b) has a W of $128 \mu \mathrm{m}$ (c) has $a W$ of $0.5 \mathrm{~mm}$ where the texture, damage and stress distributions are displayed, respectively (Papanikolaou et al. 2017, under review).

Figure 8 shows the damage and stress distributions for initial crack widths of $16 \mu \mathrm{m}, 128 \mu \mathrm{m}$, and $0.5 \mathrm{~mm}$ at $D=2.85$. Though there is a transition from notch-located crack initiation to bulk material crack nucleation, this behavior occurred at a disorder ratio $\mathrm{R}_{\mathrm{G}}$ of 0.8 where the material fluctuations are the largest on the tested interval. When the fractal surface roughness is increased, this fracture behavior occurs at disorder ratios of approximately $\sim 0.2$, much lower in the tested 
interval. At a disorder ratio of 0.8 for the higher fractal dimension, the specimens produce multiple nucleation points with significantly more damage. Nevertheless, at lower fractal dimension $\mathrm{D}=$ 2.85 , the crack propagation is quite brittle with almost no branches of secondary cracks forming.

The characteristic effect that the fractal dimension has on the disorder distribution is that it allows for larger quenched fluctuations in the critical strain energy release rate. In turn, secondary crack formation can be seen as fractal dimension is increased. Moreover, due to the fact that the large fractal dimension causes spatially sharper extremes in the disorder distribution, damage is much more likely to occur in areas away from the notch. The fractal dimension is crucial in bypassing the mesh discretization resolution, which naturally leads to artificial stress concentrations near the engineered ellipsoidal notch.

Next, we consider how the variance parameter, G, effects material disorder. Before we look at how disorder alters fracture, it is prudent to understand, fundamentally, what is being altered when discussing disorder. As stated, we utilize the $\mathrm{W}-\mathrm{M}$ function to create a fluctuating distribution in the critical strain energy release rate. The critical strain energy release rate is a parameter used in DAMASK to determine if an RVE meets the criteria for damage to occur.

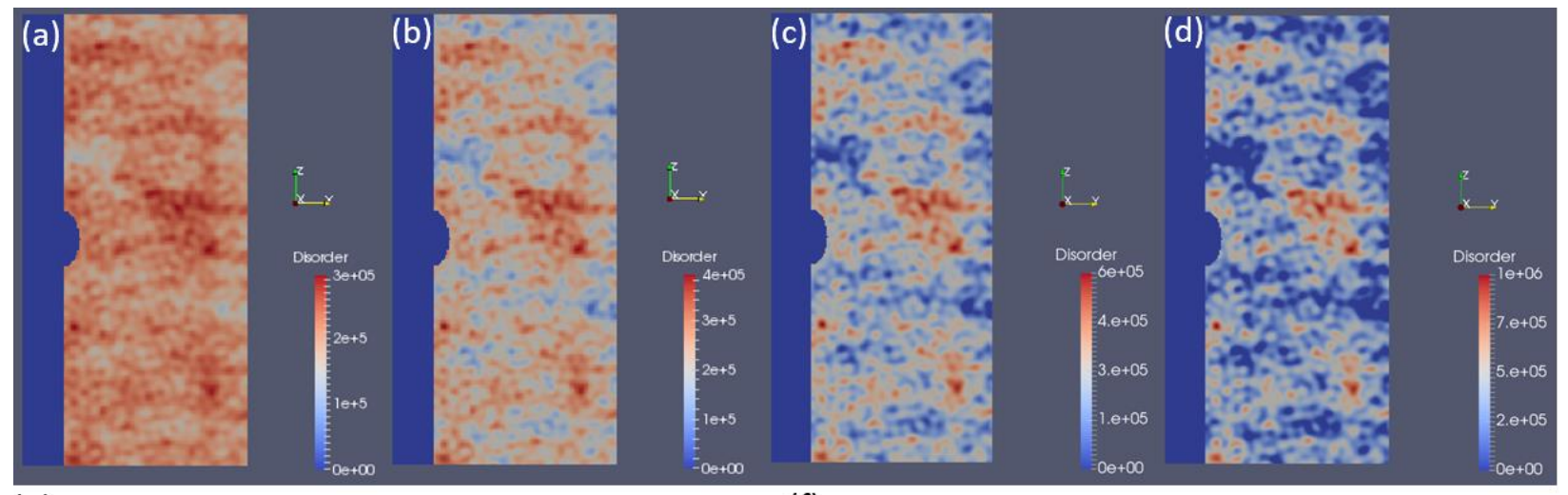

(e)

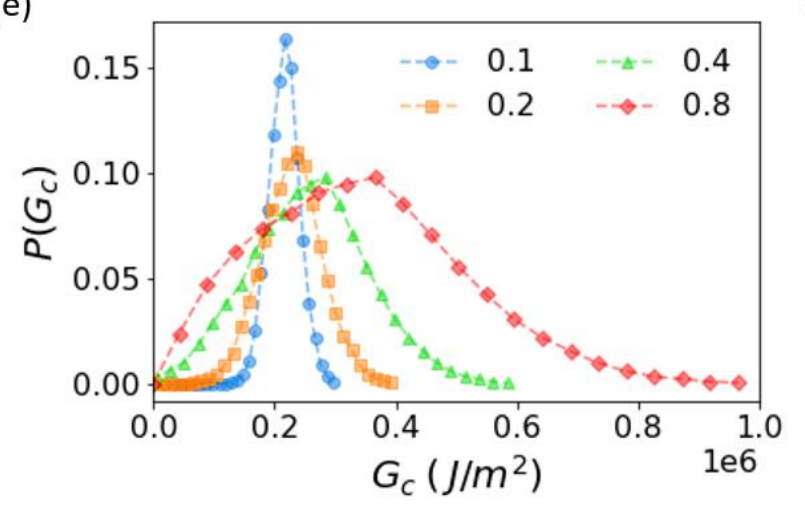

(f)

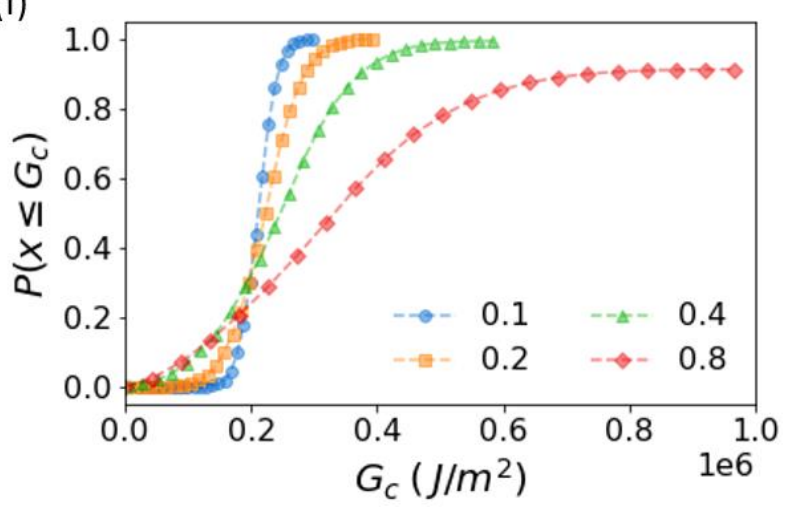

Figure 9: Disorder distribution for local fracture tendency and spatial profiles:: The progression of the disorder ratio, $R_{G}$, for $W=0.125 \mathrm{~mm}$ with a system size of $L_{x}=8 \mu \mathrm{m}, L_{y}=0.5 \mathrm{~mm}$, and $L_{z}=1.0 \mathrm{~mm}$ where (a) has a disorder ratio of 0.1 , (b) has a disorder ratio of $0.2,(c)$ has a disorder ratio of $0.4,(d)$ has a disorder ratio of $0.8,(e)$ and $(f)$ are the probability density 
function ( $p d f)$ and cumulative distribution function (cdf), respectively, of the fluctuating phase field energy parameter of each node vs. the phase field energy parameter (Papanikolaou et al. 2017, under review).

Figure 9 depicts a progression of the increasing $R_{G}$. As the disorder distributions are created at lower disorder ratios, the fluctuations in the critical $\mathrm{J}$-integral value, $\mathrm{J}_{\mathrm{Ic}}$, will be relatively small. But at higher disorder ratios, the variation in the critical strain energy or material fracture toughness will be much higher.

Physically, the $R_{G}$ values from the simulations are meant to represent the microstructurally tough and weak areas in the material. We choose the average critical strain energy of the material to be a value of $200 \mathrm{~kJ} / \mathrm{m}^{2}$, rendering the sample quite brittle, a value typically used for NiAl. If the disorder distribution ratio $\mathrm{R}_{\mathrm{G}}$ is 0.1 , then the variance in the surface toughness occurs in the interval of $[1 \mathrm{e} 5,3 \mathrm{e} 5] \mathrm{J} / \mathrm{m}^{2}$, so the maximum and minimum toughness can only be $300 \mathrm{~kJ} / \mathrm{m}^{2}$ and $100 \mathrm{~kJ} / \mathrm{m}^{2}$, respectively. The distribution for $\mathrm{R}_{\mathrm{G}}=0.1$ only show small fluctuations. For $\mathrm{R}_{\mathrm{G}}=0.8$, the critical strain energy release rate values lie on the interval $[0.0 \mathrm{e} 0,1.0 \mathrm{e} 6] \mathrm{J} / \mathrm{m}^{2}$ so the maximum and minimum toughness can be $1 \mathrm{MJ} / \mathrm{m}^{2}$ and $0 \mathrm{~J} / \mathrm{m}^{2}$, respectively, displaying sharper, larger fluctuations. It is observed in panel (e) that the width of the distributions for disorder of 0.4 and 0.8 would extend well into the negative values for critical strain energy release rate. The simulation assigns an absolute minimum value to RVEs which would have been negative in the distribution, according the W-M function. Furthermore, panel (e) refers to the histogram of the critical strain energy release rate values. Higher $\mathrm{R}_{\mathrm{G}}$ curves appear to be normally distributed close to the mean critical strain energy release rate value mentioned previously.

\section{iii. Width of Notch and its Effects on Crack Initiation and Propagation}

Fracture that initiates from the notch, typically, exhibits elastic characteristics which lie in the brittle regime. In the next series of simulations, we show that the notch width can still have a deterministic effect on the damage evolution in the sample. Under Mode I loading, the sample will show stresses concentrate at the notch tip. However, as the tip curvature softens, the stresses at the tip dissipate out into areas ahead of the notch. The transition in crack formation location is highly dependent on disorder strength, but it can still be manipulated through notch curvature at a specific disorder ratio range $\left(R_{G} \sim 0.19\right.$ to 0.24$)$ which is why the notch is used as a tool to study fracture. 


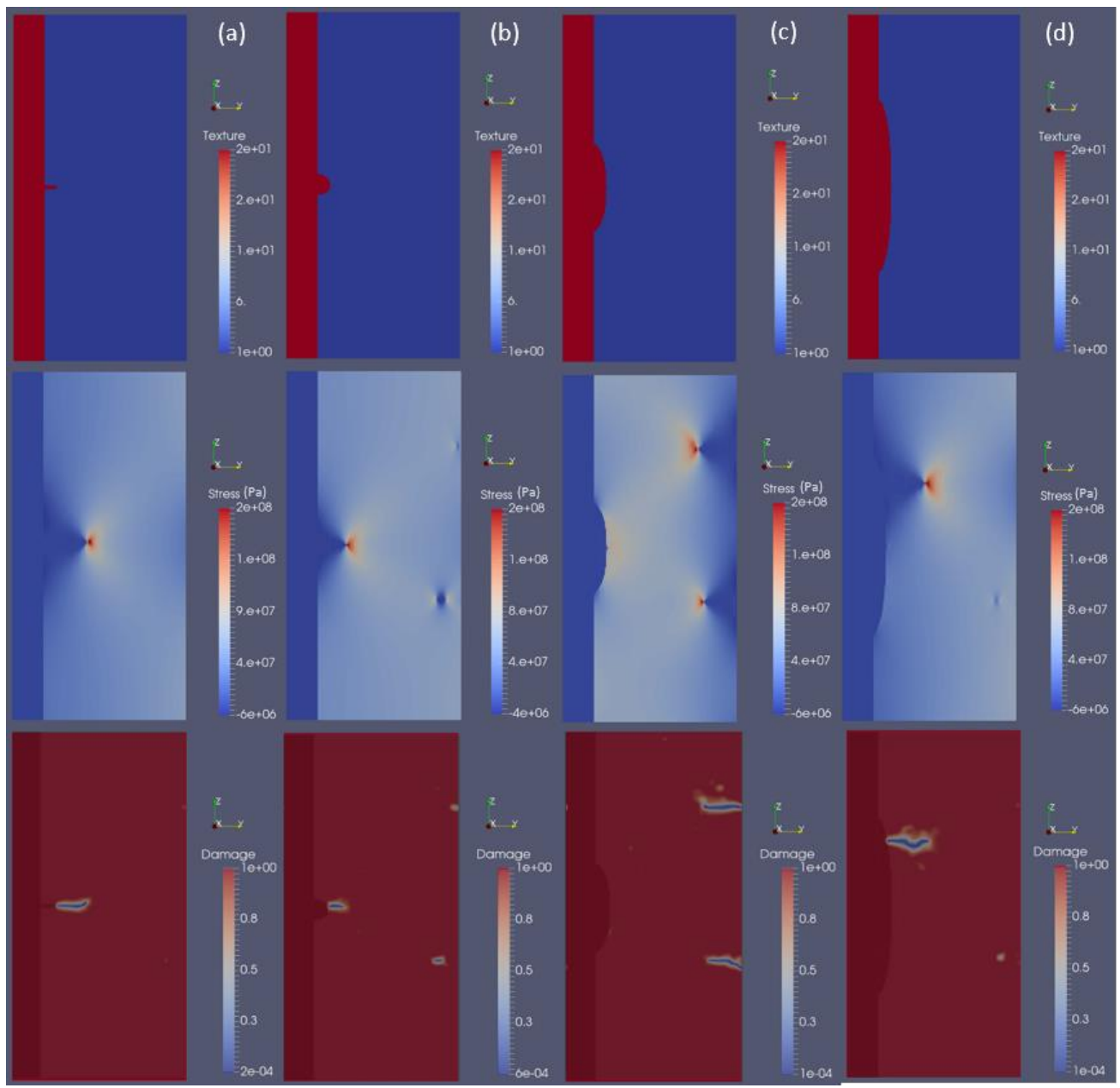

Figure 10: Effect of notch width on crack initiation in a disordered crystal:: Simulations of system size of $L_{x}=8 \mu m, L_{y}=0.5$ $\mathrm{mm}$, and $L_{z}=1.0 \mathrm{~mm}$ where $D=2.995, R_{G}=0.2$, and (a) has a W of $16 \mu \mathrm{m}$ (b) has a W of 64 um (c) has a Wof $0.25 \mathrm{~mm}$ (d) has a $W$ of $0.5 \mathrm{~mm}$ where the texture, stress and damage distributions are displayed (top, middle, bottom), respectively (Papanikolaou et al. 2017, under review).

Figure 10 shows the crack propagation behavior of disorder strength, $R_{G}=0.2$, with respect to the various notch widths of $16 \mu \mathrm{m}, 64 \mu \mathrm{m}, 256 \mu \mathrm{m}$, and $512 \mu \mathrm{m}$. The load case and disorder distributions for these samples were held constant across the simulations. At lower disorder ratios that do not lie within the range for the transition, the crack forms at the notch tip. When the disorder ratio enters the range of the transition, notch width will affect the crack formation location. As seen in Figure 10, the disorder ratio is 0.2 and the transition from notch-based fracture to disorderdriven fracture occurs somewhere between the interval of 16 microns to 64 microns. As the disorder ratio increases, the transition will occur at smaller crack widths because the notch will have less of an effect on crack formation. 


\section{iv. Fracture Characteristics for a Fixed-Dimensioned Notch with Increasing Disorder}

The cornerstone of this thesis is the ability to quantify and characterize the effect of microstructural disorder on fracture. We use the notch as a tool to promote stress concentration at the tip of the notch curvature because fracture occurs in a very predictable way with this geometry. Interestingly enough, we observe a transition in fracture behavior from brittle to quasibrittle.

Similar to other works (Bazant et al. 1998 and Alava et al. 2008), there is a brittle to quasibrittle transition in the fracture behavior. Quasibrittle fracture in the context of this thesis is when cracks nucleate in the material bulk at location with locally low critical strain energy release rate values. We investigate this transition and other features of the fracture that occur as a result of the increasing microstructural heterogeneity in terms of measurable quantities like damage, average stress and maximum stress. 


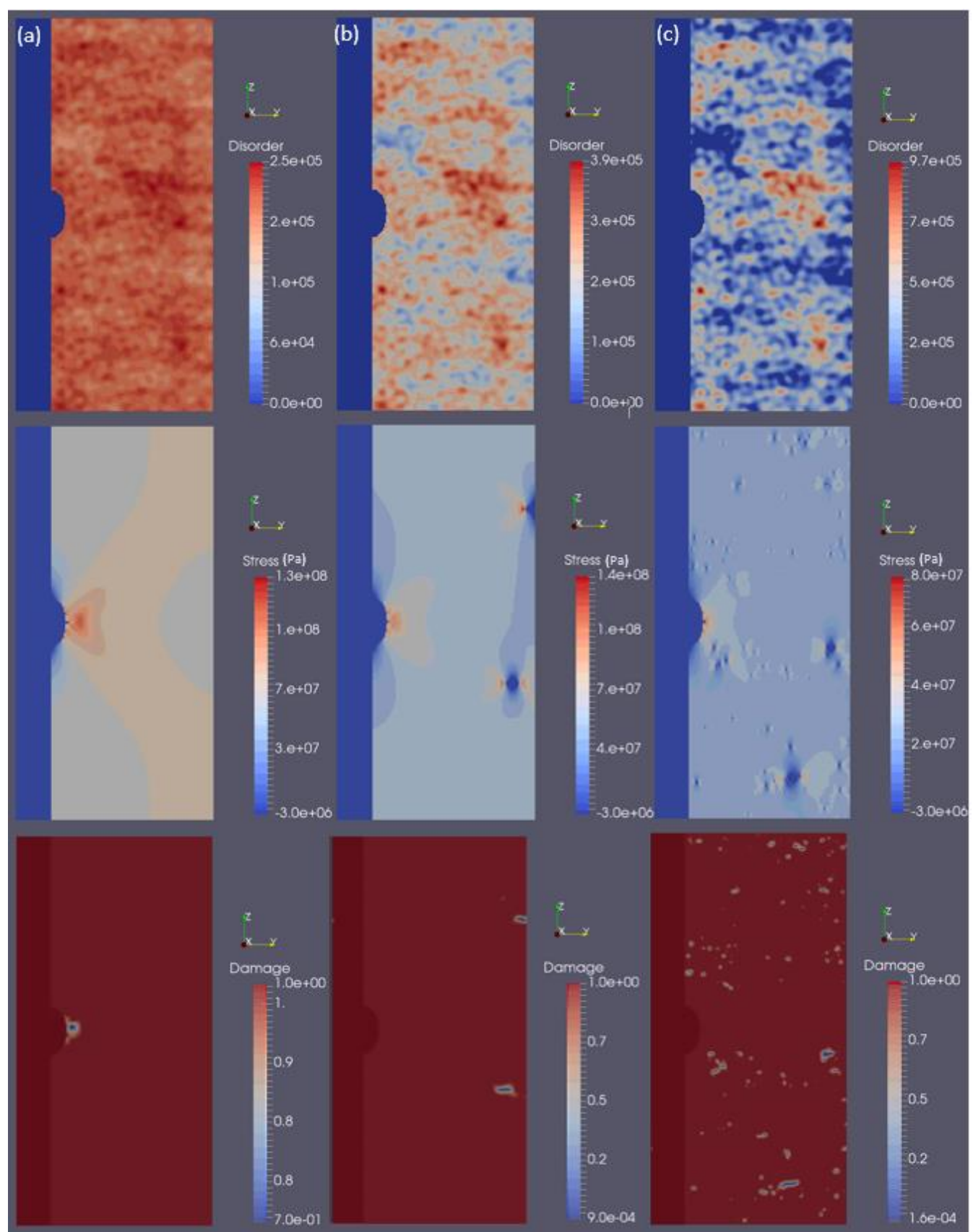

Figure 11: Effect of increasing disorder strength on crack nucleation: Simulations of system size of $L_{x}=8 \mu \mathrm{m}, L_{y}=0.5 \mathrm{~mm}$, and $L_{z}=1.0 \mathrm{~mm}$ where $D=2.995, W=0.125 \mathrm{~mm}$, and (a) has a $R_{G}$ of $0.05(b)$ has a $R_{G}$ of $0.2(c)$ has a $R_{G}$ of 0.8 where the disorder, stress and damage distributions are displayed (top, middle, bottom respectively), respectively (Papanikolaou et al. 2017, under review).

Figure 11 exemplifies the trivial and expected effect disorder has on fracture. At low disorder strengths (as seen in panel a where disorder ratio is 0.05), crack formation initiates at the notch tip. As the disorder ratios increases to 0.2 and 0.8 (panels b and c, respectively), the crack nucleates in the bulk of the material. The increased fluctuations in the critical strain energy release rate clearly show that the location of damage moves away from the notch. As it progresses further to a disorder 
ratio of 0.8 , there are multiple points of nucleation where damage locally occurs at points corresponding to RVEs with low critical strain energy release values.

Considering the crack growth phase of fracture, for low disordered systems, the crack forms at the notch and propagate relatively straight through the sample. As the disorder increases toward the transition, the crack path becomes highly dependent on the microstructural disorder. Crack growth in a homogenous material propagates from the notch in a straight line to the other lateral edge of the sample, similar to low disordered samples. As the disorder increases, before the transition away from the notch, the crack path deviates from that straight line because the disorder distribution begins to contribute more to the path and damage evolution in the sample. After the transition, the crack path is purely driven by microstructural disorder as observed in figure $11 \mathrm{~b}$. Just after the transition, crack initiation occurs in the bulk of the material at multiple locations on the sample while stress accumulates at the notch tip and the nucleation points. Further away from the transition, the fluctuations in the disorder become more extreme and, as a result, damage occurs across the sample in figure 11c. Not only do cracks nucleate in the bulk of the material at multiple locations, they propagate toward areas of low $G_{c}$ and other areas where damage has already occurred. There is a transition from notch-driven crack initiation to bulk disorder crack nucleation.

In order to understand this transition, it is important to focus on how the damage evolves in terms of the damage and stress distributions. For the purpose of this, we look at a sample with $W$ $=128$ microns and $R_{G}=0.8$ at different timesteps throughout the simulation. 

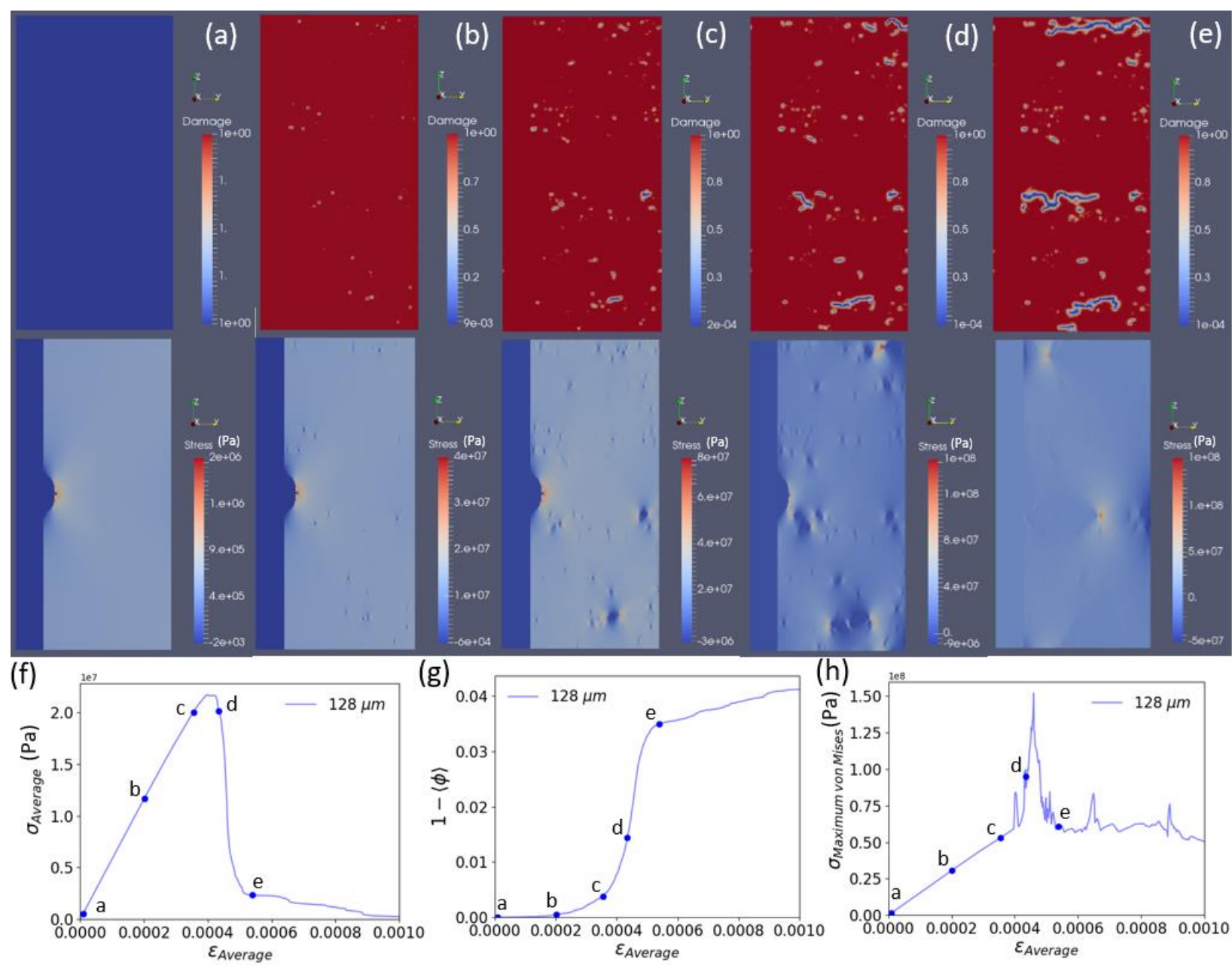

Figure 12: Stress and damage progression in a disordered, notched, crystalline sample: Simulations of system size of $L_{x}=8$ $\mu \mathrm{m}, L_{y}=0.5 \mathrm{~mm}$, and $L_{z}=1.0 \mathrm{~mm}$ where $D=2.995, W=0.125 \mathrm{~mm}$, and $R_{G}=0.8$ where $(a)$ is the undamaged phase, $(b)$ is the early damage phase, $(c)$ is the late damage accumulation phase, $(d)$ is the multiple crack nucleation event, and (e) is the crack propagation where the damage and stress distributions and are displayed (top, middle), respectively, and ( $f$ ), ( $g$ ), and (h) are the average stress ( $\mathrm{Pa})$ vs. strain, average damage vs. strain, maximum von Mises stress (Pa) vs. strain (bottom) respectively, where the five locations are identified on the curves (Papanikolaou et al. 2017, under review).

Figure 12 shows the fracture behavior as the load case progresses. We start with the sample in an undamaged state (panel a). During this phase, the sample shows no damage. We see stress, likely due to elastic deformation concentrating at the notch tip. However, as the loading continues, the microstructural disorder causes stresses to focus at locally low $G_{c}$ values which correspond to inclusions in the sample (panels b and c). As loading progresses further, we observe multiple nucleation points of cracks where enough damage has occurred and the phase field energy, $\phi$, for these RVEs are zero. Another observation at this event is that the stress begins to concentrate at the propagating cracks' tips and the inclusions that accumulated stress at the beginning of the 
simulation appear to be reduced to residual stress values. These areas of locally low $G_{c}$ values are determined by the material properties and the statistical modelling of the $\mathrm{W}-\mathrm{M}$ function.

\section{v. Quantification of Disorder in terms of the Average and Maximum Stress}

It is important to analyze the stress and damage data as a function of the load case. The simulations are processed using a python script that calculates: average stress and maximum stress of the distribution at each load step. These simulations were made varying the notch width and the disorder ratio across the defined intervals. Analyzing the simulations, even outside the transitional region, the notch still contributes to the character of the facture. The results of these simulations are produced below and the evidence of this transition is obvious in these series of plots. In the context of this thesis, brittle fracture is observed at lower disorder when the crack initiates at the notch and propagates through the sample in a linear fashion. Furthermore, damage in the phase field is localized to the region around the crack tip. As disorder increases, multiple cracks nucleate in the bulk of the sample and the stress profile developed is more characteristic of quasibrittle fracture. This behavior is consistent in all of the findings for the data and can be observed through analyzing the maximum and average stress profiles. 

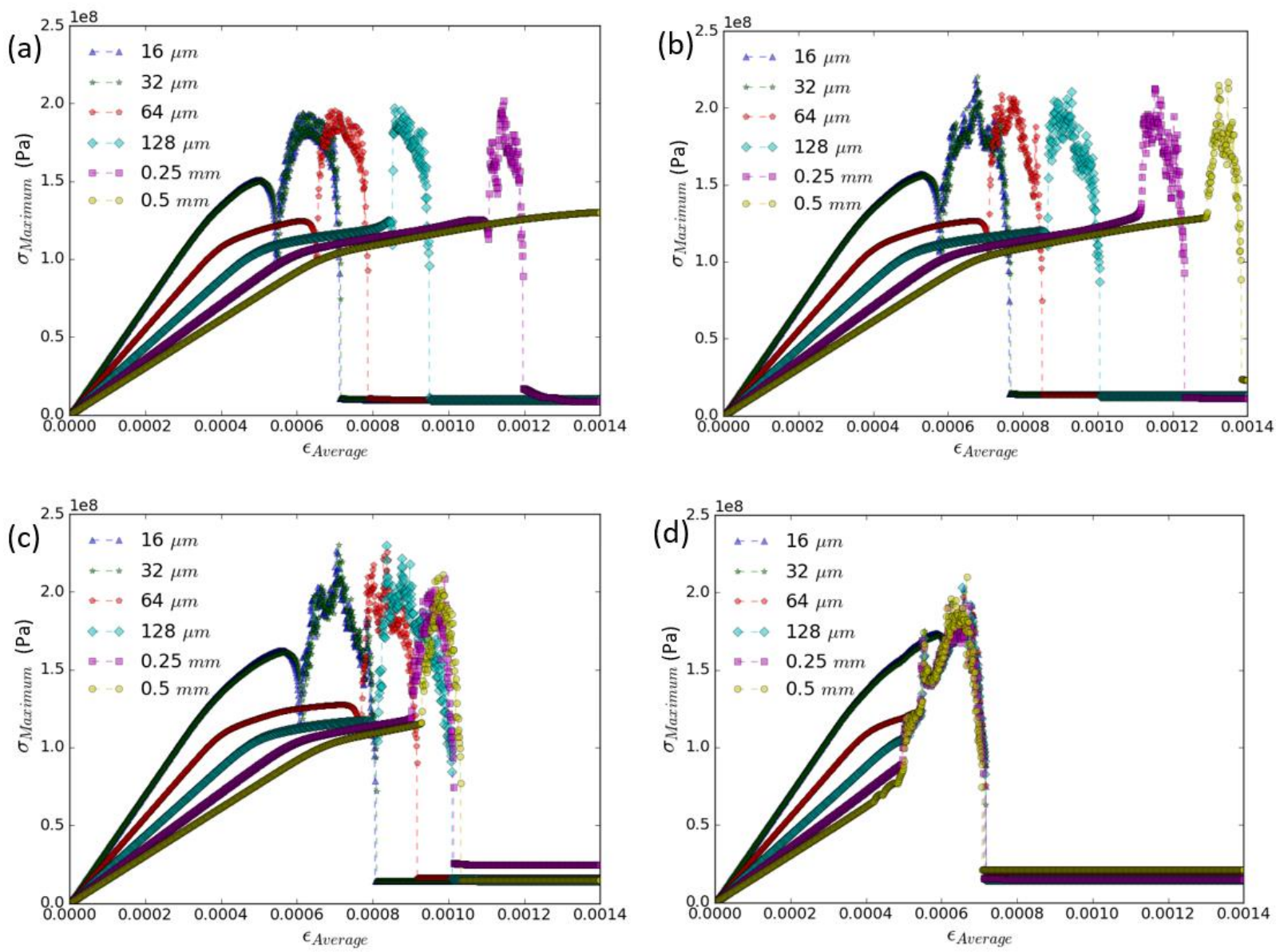

Figure 13: Effect of disorder on maximum stress: The maximum stress of samples of $L_{x}=8 \mu \mathrm{m}, L_{y}=0.5 \mathrm{~mm}$, and $L_{z}=1.0 \mathrm{~mm}$ where $D=2.995$ as a function of the average strain with respect to $R_{G}$ where $(a)$ has $a R_{G}=0.0$, (b) has a $R_{G}=0.1$, (c) has a $R_{G}=0.2$, and (d) has a $R_{G}=0.4$ (Papanikolaou et al. 2017, under review).

Figure 13 is a plot of the maximum stress in the samples with various notch widths from 16 to 512 microns and disorder from 0.0 to 0.4 disorder ratio. During loading in the pre-cracking regime, these plots show the maximum stress found in a single RVE compared to the whole system. Typically, this RVE is located at the tip of the notch in homogeneous or low disordered samples. For highly disordered samples, this RVE is located in the material bulk at an area with an extremely low $G_{c}$ value. During loading, low disordered samples with small notch width have higher slopes in the maximum stress profile. This corresponds to a classic elastic response to the notchedgeometry of the samples. This observation is consistent for all simulation before crack formation. At higher disorder strengths, the onset of crack formation occurs much earlier in simulations as seen in panel (c) and (d). We even see the same delay of crack formation behavior observed in the average stress profiles for low disorder and large notch widths. After the formation of the crack, the stress jumps to some very large values, almost $200 \mathrm{MPa}$. Furthermore, these jumps occur 
similarly across all notch widths in the various disorder strengths. There are several reasons this occurs. First, the simulation is approximating a single discretization unit as the notch tip where, physically, there is a singularity due to a very sharp tip. Second, the maximum stress is calculated from the crack tip which is experiencing plastic strain. Therefore, the maximum stress will be much larger at the crack tip due to the plastic regime of fracture.
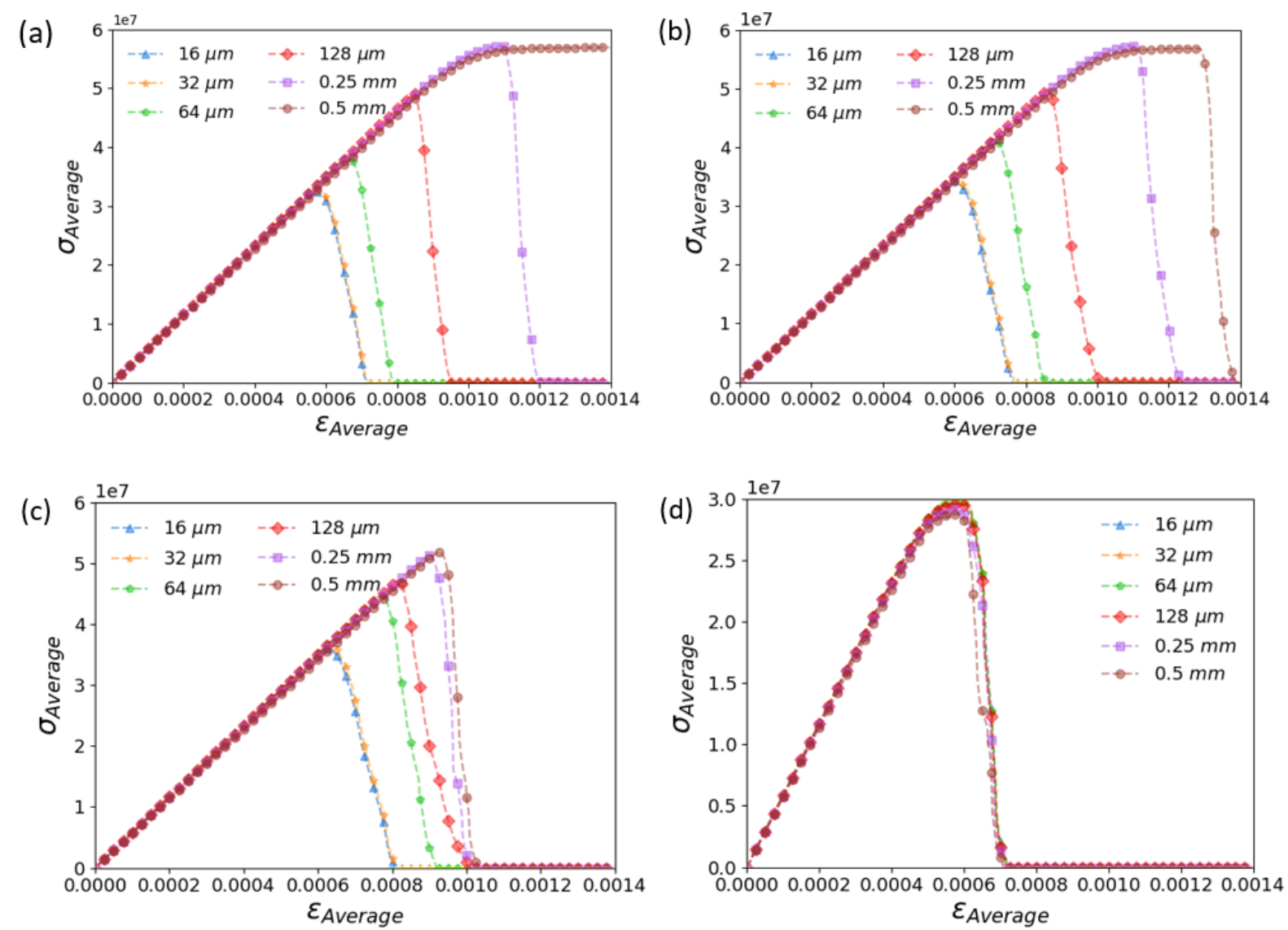

Figure 14: Effect of disorder on average stress: The average stress of the specimens of $L_{x}=8 \mu \mathrm{m}, L_{y}=0.5 \mathrm{~mm}$, and $L_{z}=1.0$ $m m$ where $D=2.995$ as a function of the average strain with respect to $R_{G}$ where $\left(\right.$ a) has a $R_{G}=0.0,(b)$ has a $R_{G}=0.1,(c)$ has a $R_{G}=0.2$, and (d) has a $R_{G}=0.4$ (Papanikolaou et al. 2017, under review).

Figure 14 is a plot of the average stress in the samples with various notch widths from 16 to 512 microns and disorder from 0.0 to 0.4 disorder ratio. These observations are analogous to ones seen in the average stress profiles. At low disorder strengths, fracture in samples can be characterized as brittle (Figure 14a), observing linear stress increases until fracture and immediate failure for crack widths of 16 to 128 microns. Even though crack initiation occurs at the notch in Figure 14a for the 256 and 512 micron notch widths, the stress profiles begin to exhibit plastic qualities due to the delayed failure after the linear stress increase from such large notch widths. With the addition of disorder (Figure 14b), this plastic behavior begins to have less of an effect on 
the samples as the delay to failure is shortened at large notch widths. Continuing to increase disorder in the simulations, the samples begin to exhibit similar fracture characteristics independent of notch contribution as seen in Figure 14c where the samples with crack widths of 256 and 512 microns display very similar average stress profiles compared to the other notched samples at the disorder strength of 0.2. At a disorder strength of 0.4 (Figure 14d), we observe the other side of the transition where fracture is characterized as quasibrittle. Fracture is similar and consistent across all of samples independent of notch width. Let's take a closer look at the fluctuations in the average stress profiles with increasing disorder for a fixed-dimensioned notch.
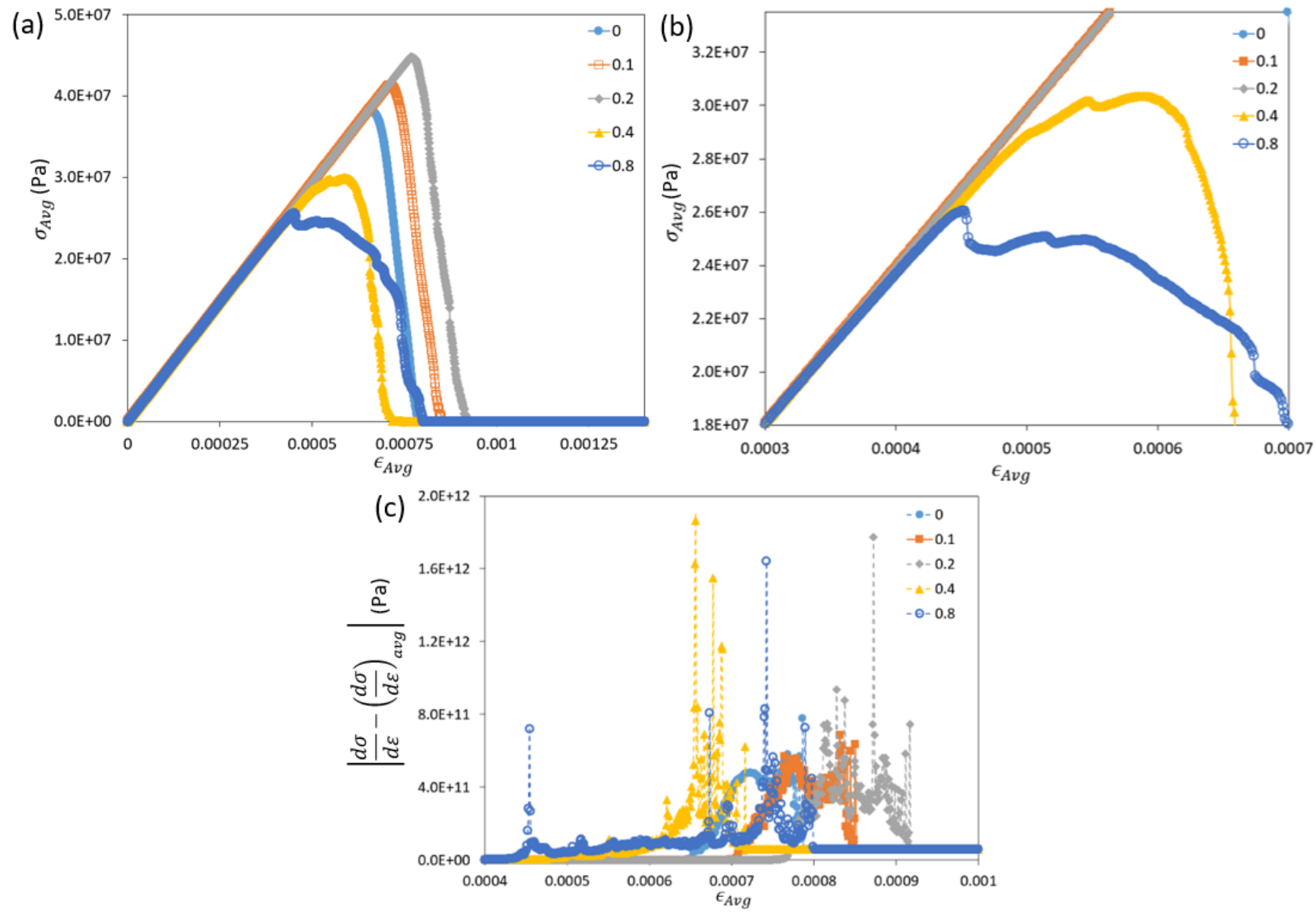

Figure 15: Quantification of the fluctuations at crack nucleation and growth of average stress: Simulations of sample size $L_{x}=8 \mu \mathrm{m}, L_{y}=0.5 \mathrm{~mm}$, and $L_{z}=1.0 \mathrm{~mm}$ where $D=2.995$ and $W=64 \mu \mathrm{m}$ as a function of the average strain where (a) is the average stress vs. average strain, $(b)$ is the zoomed-in view of the fluctuations for disorder strengths 0.4 and 0.8 , and (c) is the difference in the rate of change of the average stress and the initial rate of change of linear increase in average stress vs. average strain (Papanikolaou et al. 2017, under review).

Figure 15 highlights the characteristic effect that disorder has on the crack formation and growth. For the lower disorder strengths, the fracture was brittle corresponding to the standard stress profiles seen in Figure 15a. However, as we plot the average stress profiles with for the same notch width and increasing disorder, the samples with larger disordered fluctuations display more 
stochastic changes in the rate of change stress profiles (Figure 15c). The low disorder samples such as $R_{G}=0.0$ and 0.1 don't experience the same sharp jumps in the slope at crack initiation due the notched geometry and low disorder ratio. Furthermore, their rate of change increases in average stress as a function of strain are mainly focused around the crack initiation and growth region. As disorder strength increases, we observe precursor events before crack nucleation as seen in the $R_{G}$ $=0.8$ profile for around 0.00045 strain. These precursor events and crack propagations characteristics show a quantifiable difference at higher disorder strengths including longer more stochastic fracture. Given this evidence, we seek to generally characterize these observations and compare all of the samples with various notch curvatures and disorder strangths.

As we identify the brittle to quasi-brittle transition as a function of disorder strength, the stress event size is insensitive to the notch dimensions after the transition to quasi-brittle fracture. We present two plots below that we use to observe and analyze the fracture behavior. The number of events and their corresponding size were measured to classify the fracture as brittle or quasi-brittle. Brittle samples exhibit one large stress event which is consistent with observations. This event is the failure of the sample as the crack initiates at the notch and propagates through the sample. Quasi-brittle fracture consistently exhibits multiple stress events. These events are a combination of one large failure event and several smaller precursor events.
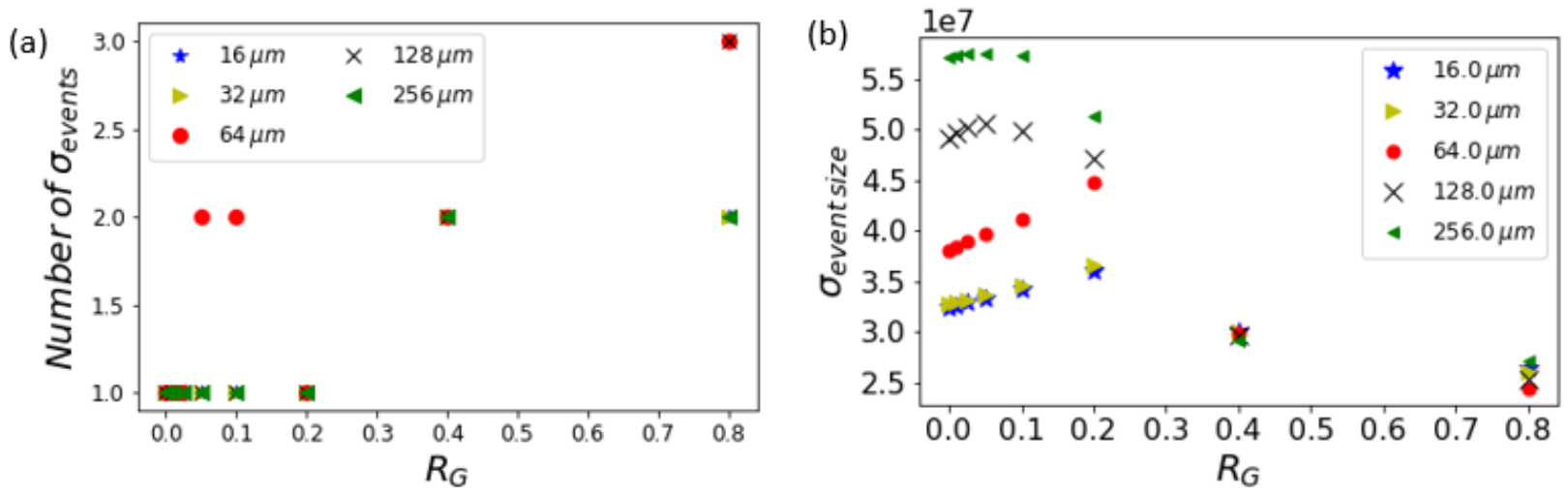

Figure 16: Significant Stress Events for Brittle and Quasi-brittle Fracture: The number and size of the stress events were measured as a function of $R_{G}$ where $(a)$ is a series of histogram curves for various $W_{C}$ and $(b)$ is a plot of the event size for various $W_{C}$ (Papanikolaou et al. 2017, under review). Note that the notches of widths: 16, 32, 128, and 256 microns only have one stress event for $R_{G} \leq 0.2$ while the notch width of 64 microns was observed to have two stress events for $R_{G}$ of 0.5 and 1.0. These events have a magnitude on the order of about $10^{5} \mathrm{~Pa}$. Also, for $R_{G}=0.4$, there are two stress events for all notch widths and both events share approximately the same event size for all notch widths.

Figure 16 shows the characteristic effect of brittle and quasi-brittle samples which can be analyzed by measuring the stress events. The overall trend for the figure: As the disorder ratio increases, the 
average stress curves transition from brittle to quasi-brittle fracture, as expected. This is indicated by observing that brittle samples with low disorder only have a single stress event during fracture where stress events are defined as significant stress drops in the average stress profile. Quasi-brittle samples are seen to have multiple stress events as seen in figure 16a. Though one stress event dominates the fracture, the precursor events still have significant stress drops that must be considered. Furthermore, in Figure 16b, the stress events demonstrate clear insensitivity in the maximum average stress at similar strain values. We observe this by noting that the significant stress drops converge to a small range of values close to $30 \mathrm{MPa}$ at $R_{G}=0.2$ and, approximately, $25 \mathrm{MPa}$ at $R_{G}=0.8$. While trivial that crack nucleation depends on the competition between local variations in material properties and the notch, it should be noted. As they approach this transition, the disorder introduces stochastic fracture effects in the model material. 


\section{Active Disordered Systems: Bidirectional flow of binary self- propelled particles}

\subsection{Literature Review}

A diverse range of systems can be effectively modelled as groups of interacting particles coupled with external drives of opposite sign in a channel configuration. These systems can be considered with a quenched disordered configuration and can exhibit a rich variety of dynamic behaviors (Schmittmann and Zia 1993, Helbing et al. 2000, and Dzubiella et al. 2002) from a jammed state to laning state (Reichhardt and Reichhardt 2016). Analogous to these binary systems are swarms of insects (Couzin and Franks 2003), flocks of birds, and large crowds of people (Helbing et al. 2000 and Moussaid et al. 2009 ). These transitions from a pinned or jammed state can be elastic where individual particles keep their same neighbors moving through the system or plastic where individual particles gain new neighboring particles to interact with (Bhattacharya and Higgins 1993, Reichhardt and Reichhardt 2016, and Reichhardt and Reichhardt 2018). Typically, the classification of behavior as elastic or plastic is characteristic of the ordering of the particles in the structure: The more disordered the structure, the more plastic the systems is. When particles obtain a high mobility, they form noncolliding chains and the elastic system transitions to a more ordered structure. At higher drive forces, a transition from plastic flow to elastic flow can be observed as the structure of the system moves from a mixed, disordered state to a laning, ordered one. The translation is attributed to increases in the ordering of the system which produces a definable change in the structure factor (Moon et al. 1996, Pardo et al. 1998 and Olson et al. 1998). Depending on the parameters used, these phase transitions can present in a continuous or first order manner (Bhattacharya and Higgins 1993, Marchetti et al. 2003, and Reichhardt and Reichhardt 2016).

In most systems, particle-particle interactions are modelled as a repulsive smooth potential. These potentials can be considered long-range interactions where particles interact over distances or short-range interactions where a hard surface particle approximation is used. Both interactions yield very different system responses and transitions. For example, long-range interactions can cause systems to form ordered solid states even at low densities, while short-range interactions would express more fluid-like systems at low densities and pinned configurations at higher packed systems. Our model uses a short-range interaction to approximate a hard surface with spring-like, linear potential. However, this is not the only motion-contributing factor. Characteristic of large 
crowds, there is a unique motion to each individual person. In condensed matter, this characteristic is, typically, described as activity. Adding activity to a non-active system can cause pinned structures to break apart and increase the mobility of the system. When phase-separated, non-active states become active, they are susceptible to jamming or clogging through a freezing-by-heating effect (Helbing et al. 2000).

Active matter systems are comprised of self-propelled particles (SSP) which consume forms of energy to produce directed motion. In recent models, active disks often obey run-and-tumble dynamics where a defined parameter such as runtime allows for an individual particle to travel in a random direction for a number of timesteps in the simulation, then the particle chooses another direction and repeats the process for the duration of the simulation. This unique motion of the particles can contribute to several of the phase transitions in the model when drive force is low enough (Tailleur and Cates 2008, Cates and Tailleur 2013, Reichhardt and Reichhardt 2015, and Bechinger et al. 2016). This gives rise to a natural competition between the individual activity of the particles and the overall mobility of the system.

In this study, we analyze the dynamic phase transitions of a binary system of oppositely driven active run-and-tumble particles. The simulation begins with quenched disorder characteristics where the particles use several forces to interact with each other and contribute to their motion within the system. The external drive force $\boldsymbol{F}_{d}^{i}$ varied on the interval $[0.0,12.0]$ and the motor force $\boldsymbol{F}_{m}^{i}$, governed by the runtime $\tau_{r}$, is varied on the interval [20 to 2000]. The system density, $\phi$, is varied from $[\sim 5 \%, \sim 85 \%]$. In the following sections, we explain how the system is constructed (see Section 3.2), what the individual contributions to the particle's motion are (see Section 3.2), the various phase transitions observed which characterize the systems steady state behavior (see Section 3.3). Also, this thesis addresses how these transitions change based on the parameters tuned: runtime, drive force, and system density where one sees observable drops in the average velocity and order of the structure (see Section 3.3). 


\subsection{The Model}

We perform active matter simulations using a simple molecular dynamics model. Because this is a two-dimensional simulation, the technical term for the objects used is disks, given that they do not have a third dimension of thickness. However, for all intents and purposes, they can be considered particles even though the term implies a three-dimensional shape. We can vary the simulation's number of disks/ particles from $N=2$ to $N \sim 1450$ which is a system density, $\phi$, of $[<1 \%, \sim 87 \%]$. Exceeding this number of particles is impossible as they are round and therefore cannot occupy the square space fully. Of the simulation setup, the boundaries are periodic in the upper, lower, and lateral edges. These boundary constraints allow for a channel type flow for the particles to move within.

The system is of size $L \times L$ with $L=36$. The particles are place in the system with a random distribution. Since the system has been defined, it is pertinent to discuss the laws of motion and interaction that the particles will be defined by.

There are several forces that contribute the overall motion of the individual particles. The first defines the interaction of the various particles within the system, $\boldsymbol{F}_{p p}^{i}$. While we cannot define a hard boundary due to the limitations of using a molecular dynamics model, a hard surface interaction can be approximated using a repulsive force for the individual particles. The repulsive interaction is modelled as a spring force.

$$
\boldsymbol{F}_{p p}^{i}(r)=\Theta(2 R-d) k(2 R-d) \hat{d}
$$

Where $d$ is the distance between the centers of the particles, $\widehat{\boldsymbol{d}}$ is the displacement vector between the particles, $k=20.0$ is the spring constant, and $\Theta(x)$ is the Heaviside function. The Heaviside function is a step function which assigns a value of $\Theta(x)=0$ or $\Theta(x)=1$ depending on value of $d-2 R$ which is the distance of the two radii of the particles. If $d>2 R$, then the particles are not in contact and as a result the function, $\Theta(x)$, yields a zero value for the force. But if $d<2 R$, then the spring force is considered. We use a particle radius of $R=1.0$. Therefore, the particle density can be calculated for the system. This parameter is useful to plot various metrics against because it yields obvious, but interesting transitions in flow behavior. The system density is defined as the area the particles cover.

$$
\phi=N \pi R^{2} / L^{2}
$$


yielding a range of densities of $\phi=0.0496$ to $\phi=0.8496$ for 82 to 1402 particles, respectively. Next, a uniform external drive force, $\boldsymbol{F}_{d}^{i}$, is applied.

$$
\boldsymbol{F}_{d}^{i}=F_{d} \boldsymbol{x}^{i}
$$

where $\boldsymbol{x}^{i}=(+\widehat{\boldsymbol{x}},-\widehat{\boldsymbol{x}})$, on each disk. For half of the particles, this force is applied in the positive $\mathrm{x}$-directions and the other half experience this applied force in the opposite direction or negative $\mathrm{x}$-direction. The third force that contributes to the overall motion of the particles give them their "active" quality. The active characteristic is produce by defining the motor force, $\boldsymbol{F}_{m}^{i}$, which acts on all the particles differently. The motor force is defined by run time, $\tau_{r} . \tau_{r}$ is in units of timesteps for a given simulation. For example, if a particle has a specified runtime, then it will choose a direction randomly and travel in that fixed direction for the number of timesteps defined by the runtime before choosing another direction randomly and repeating this process for the duration of the simulation. The overall dynamic of the particles is obtained by a cumulative equation which accounts for each contribution to the motion.

$$
\eta \boldsymbol{v}_{i}=\boldsymbol{F}_{n e t}=\boldsymbol{F}_{p p}^{i}+\boldsymbol{F}_{d}^{i}+\boldsymbol{F}_{m}^{i}
$$

Where $\eta=1$ is the drag coefficient.

There are several metrics used to quantify the overall behavior of the particles. One is to measure the resulting average drift velocity per disk in the direction of the drive force to produce velocity-force curves.

$$
\langle V\rangle=N^{-1} \sum_{i=1}^{N}\left\langle\boldsymbol{v}_{i} \widehat{\boldsymbol{x}}\right\rangle
$$

Where $N$ is number of particles for one type, $\boldsymbol{v}_{i}$ is an individual particle velocity, and $\widehat{\boldsymbol{x}}$ is their respective direction. Another useful measure is of the structure of system within the simulation. We measure the density for sixfold coordinated vortices, $P_{6}$, which is a measure of order in the lattice. The equation used to solve this is:

$$
P_{6}=N_{d}^{-1} \sum_{i}^{N_{d}} \delta\left(z_{i}-6\right)
$$

Where the particle coordination number $z_{i}$ is obtained from a Voronoi tesselation and $N_{d}$ is the number of interacting particles. 


\subsection{Results/ Discussion}

The following plots and figures investigate bidirectional flows of binary self-propelled particles at different densities. We begin by looking at the phases present for low density systems (see Section 3.3.i). Next, we increase the system density and observe the presence of new phases in the system (see Section 3.3.ii). Finally, we develop a phase diagram and show how it evolves as a function of the systems parameters: run-and-tumble force, drive force, and system density (see Section 3.3.iii).

\section{i. Laning and Mixing at Low Densities}

Initial work on non-active particles expressed four dynamic phases: a jammed or pinned state, a phase separated state, a mixed or disordered state, and a laning state. However, in active systems where individual particle motion is dictated by run-and-tumble dynamics, the system displays the states mentioned above as well as several others that are similar in structure. At low densities, it is difficult to determine a phase expressed by the system, but this is a product of the sparseness of the system. At any density below $\phi=0.55$, the system is classified as a laning state because of the lack of particle collision as one type of particle moves from one side of the channel to the other. However, the mixing state is present as a result of particles moving across lanes while still maintaining their noncolliding characteristics in the system. As the density increases, the other two phase states consistently emerge in the system. 

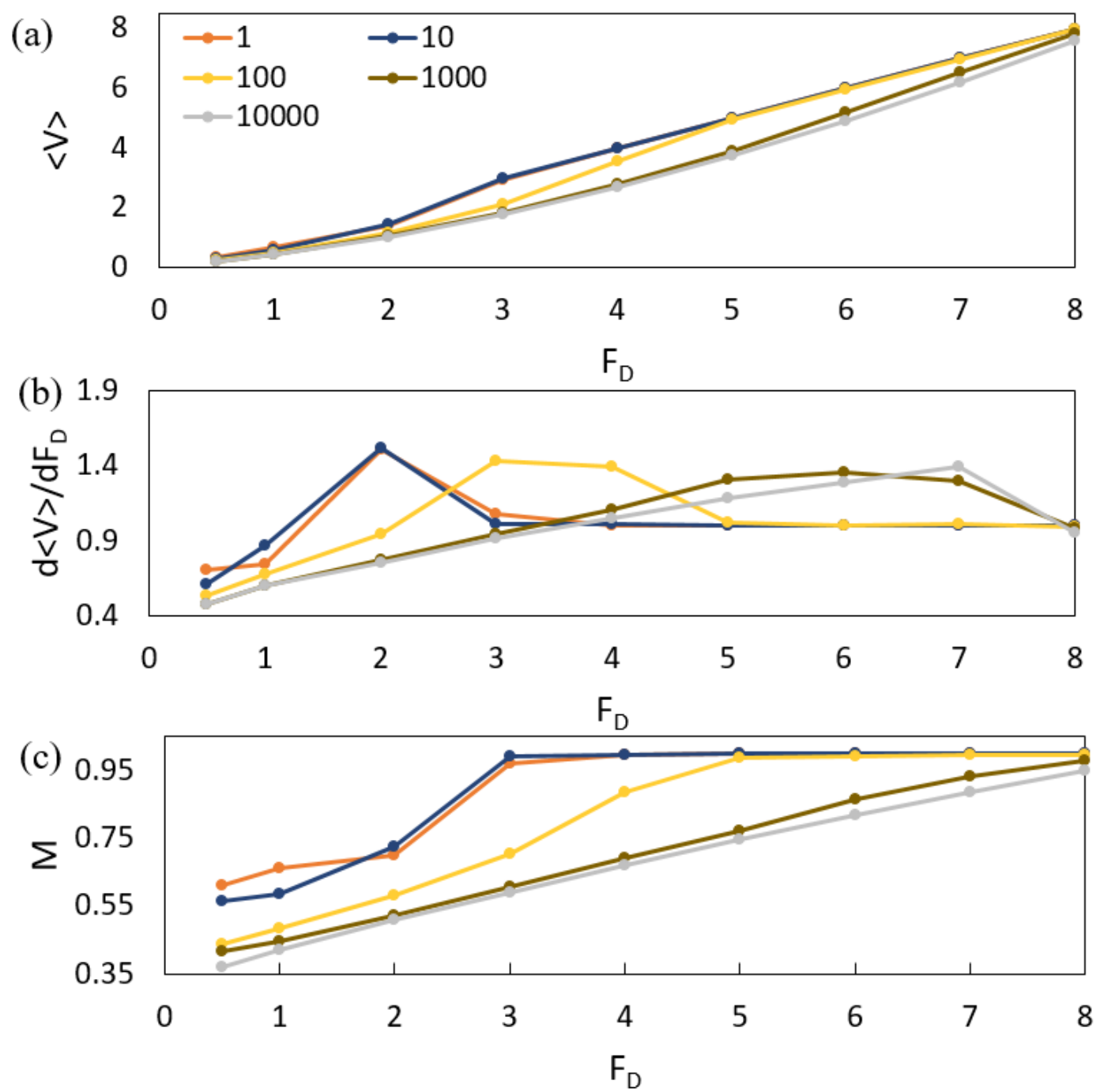

Figure 17: Average velocity and corresponding slope and mobility at low density: (a) The average velocity per particle as a function of drift in a sample of particle density of 0.55. The run-and-tumble particles have a run time of 1, 10, 100, 1000, and 10000. (b) The corresponding $d<V>/ d F_{D}$ as a function of drive force where a peak in the slope moves to higher drive force as run time increases. (c) The mobility of the particles as a function of drive force (See also Reichhardt and Reichhardt 2015 and Fig 1 in Reichhardt et al. 2018, under review).

As evidence of this transition, we plot the average velocity as a function of drive force, $\mathrm{F}_{\mathrm{D}}$, for various run time, $\tau_{\mathrm{r}}$, the corresponding slope of the average velocity, and the mobility, $M=$ $\langle V\rangle / V_{0}$. In figure $17 \mathrm{a}$, the average velocity is linear at large drive forces for $\tau_{\mathrm{r}}=1,10$, and 100 . This is corroborated by the mobility plot (Figure 17c) where the profiles for these run times approaches a value of 1 . The system is in a laning state where the particles form non-colliding chains. As run time increases, these chains break apart and the system enters into a mixing/ disordered state. The average velocity profile of this state is nonlinear and the mobility is $M<1$. Thus, the activity of the system increases by increasing the run time. 
Below a of density $\phi=0.55$ and large drive forces, a new phase emerges that is not unlikely the laning state. Similar in its structure, the smaller amounts of particles make it hard to classify it as such. It can best be described at a clustered laning state where the structure of the system allows for particles of the same type to organize into large groups compared to the number of particles in the system.
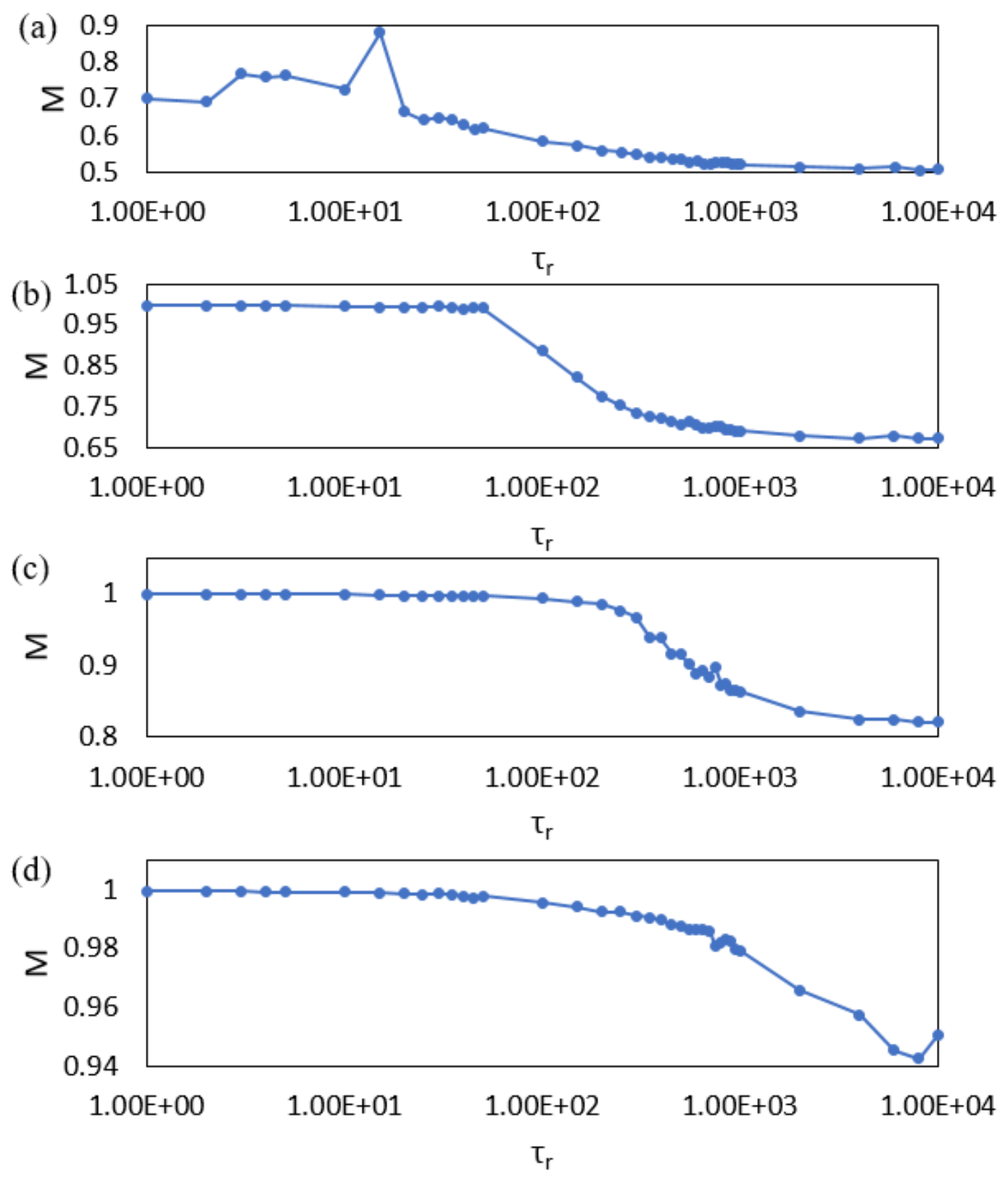

Figure 18: Mobility of various drive forces: The mobility, $M$, as a function of runtime for various drive forces for $\phi=0.55$ where (a) is a drive force of 2.0, (b) is a drive force of 4.0, (c) is a drive force of 6.0, and (d) is a drive force of 8.0 (See also Reichhardt and Reichhardt 2015 and Fig 3 in Reichhardt et al. 2018, under review). 
Mobility is best described as the normalized average velocity of one type of particle. Comparing the various mobility profiles for different drive forces, the transition occurs at higher run times as the drive force is increased. The larger the drive force, the more activity required by the particles to transition from a laning state to a mixing state. Furthermore, the transition is much sharper at lower drives and occurs more gradually as drive force is increased.

\section{ii. Phases Observed at High System Densities}

We study how the other phases appear in the system and how it evolves when the run-andtumble force and the drive force are altered. When looking at the movies produced by the simulation, it became clear that the system would have a naturally competing effect between the drive force on all the particles in the system versus the individual particle's motion which results from their runtime. For the purpose of analyzing the characteristic effect of drive force and runand-tumble force, we fix the number of particles to be 1154 , approximately $70 \%$ of the system's space. 

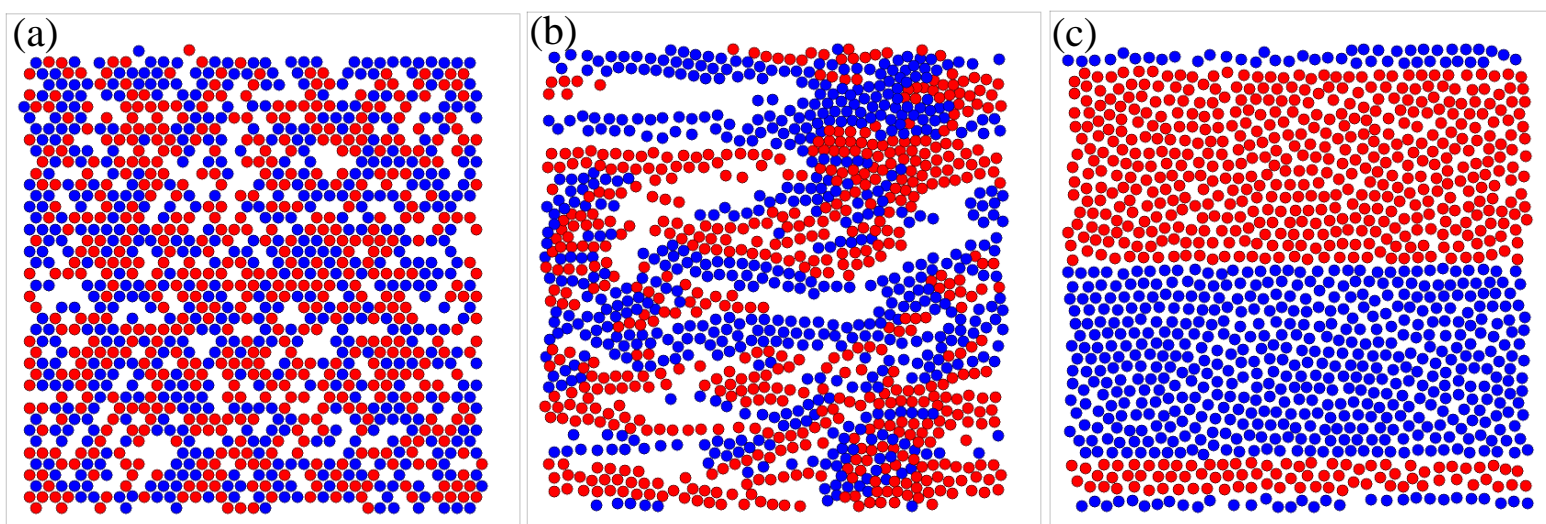

(d)

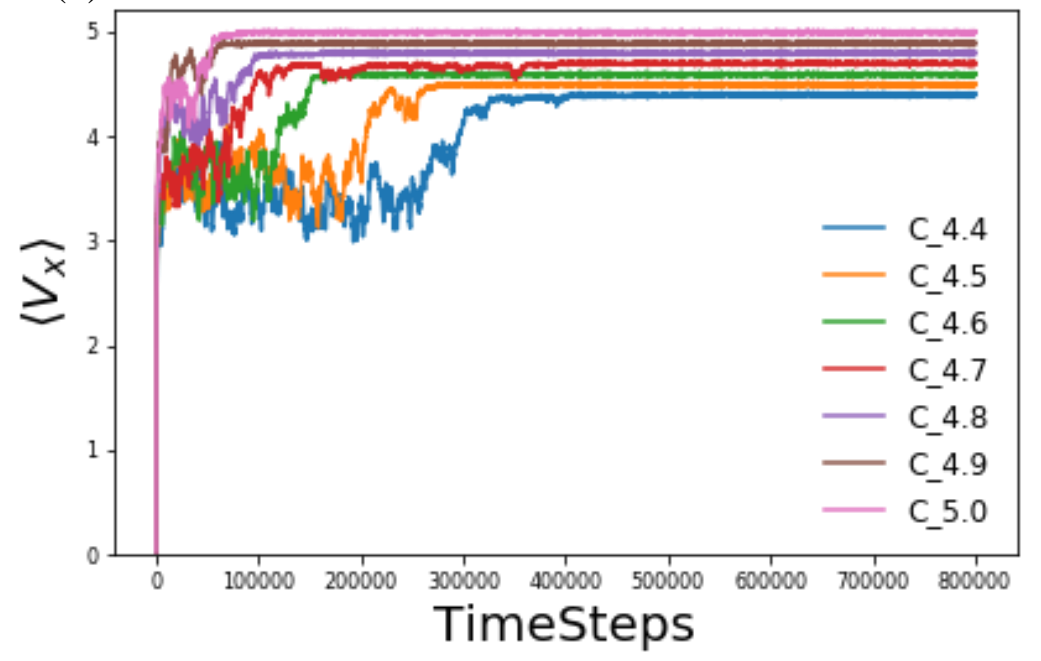

Figure 19: Characteristic effect of drive force on the system: (a) shows the system's configuration at the beginning of the simulation. (b) shows the system's configuration during the velocity fluctuations. (c) shows the system's configuration after achieving a steady state behavior. (d) Various drive forces are plotted and the number of particles was defined as 1154, a system density of about 70\%, and the runtime was fixed at 20 (See also Carbone and Cozzoli 2010, Lattuada and Hatton 2011, Reichhardt et al. 2018, under review).

Once the simulation is initialized, the particles are at a quenched disordered state, shown in Figure 19a. As the drive force is uniformly applied to all particles, they begin moving in that direction. Figure 19 shows various drive forces on a system with $\phi \sim 70 \%$ and $\tau_{r}=20$. It is observed that the lowest drive force profile takes more time to reach a steady state behavior as seen in Figure 19d. As drive force increases, the fluctuations, observed in Figure 19b, at the beginning of the simulation dampen out more quickly. At extremely high forces, the system exhibits almost no fluctuation at the beginning of its simulations. Therefore, the characteristic effect on drive force is to dampen individual particle motion, as seen in Figure 19c. This type of motion can be similar to how a flock of birds take off from a field. When they are on the ground, they are in a disordered orientation. However, when the group takes off and flies away the disorder 
seen as a result of their initial position quickly becomes a self-organized flock moving with a singular purpose. Given that the drive force is implemented in the simulations to govern the group's collective behavior, another force is used to define the random or unique behavior of individuals in a large group.

The run-and-tumble force or runtime is used to model the individual particles' unique behavior in a system. From our perspective, this unique behavior can be considered random as one does not know what the motives of the individual particles are. It has been well-document that the run-andtumble force is a sufficient approximation of individual particle motion (Tailleur and Cates 2008, Cates and Tailleur 2013, Reichhardt and Reichhardt 2015, and Bechinger et al. 2016). 

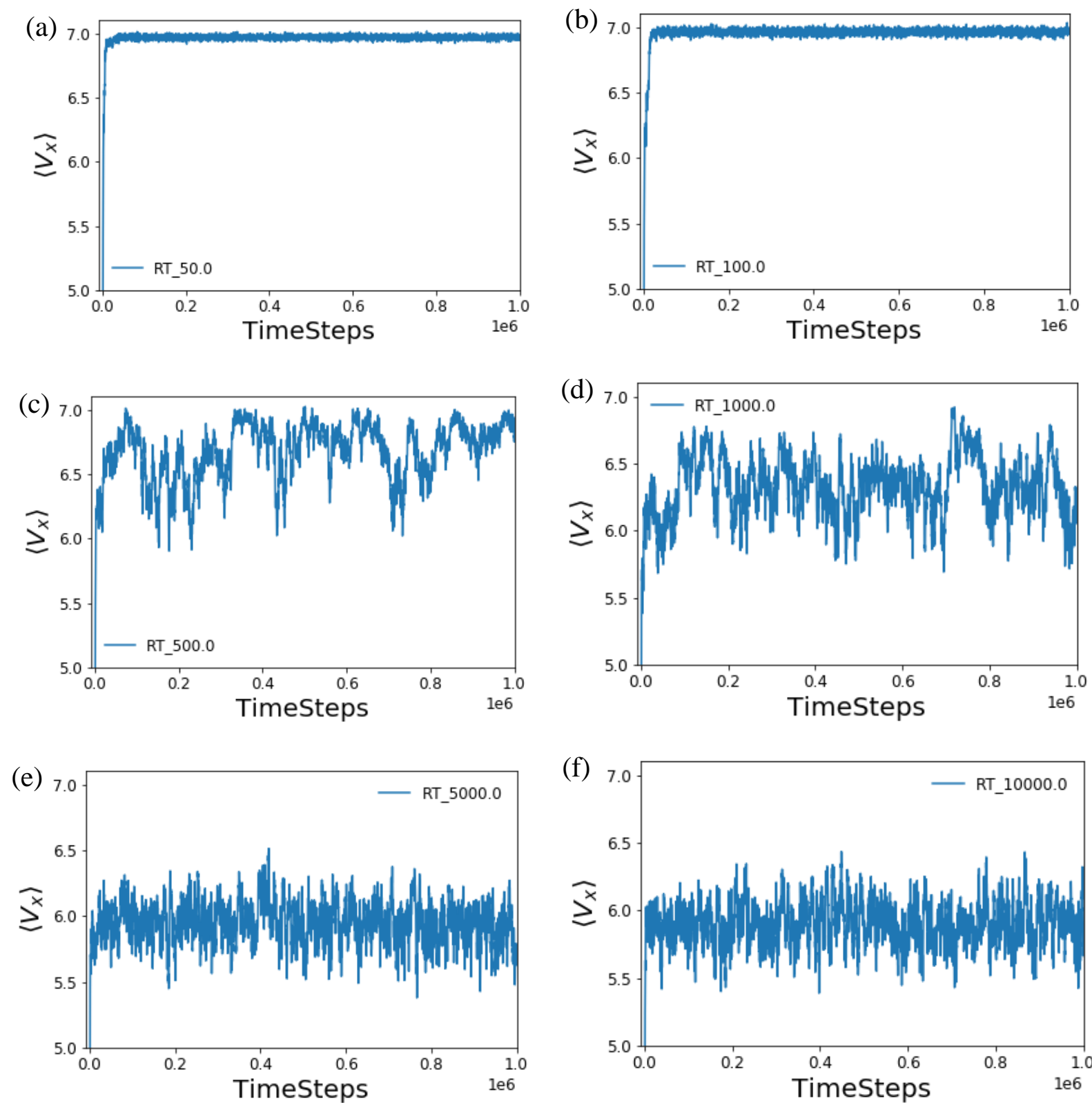

Figure 20: Characteristic effect of runtime on the system: The system's drive force and number of particles were held constant at 7.0 and 1154, respectively, and the runtime is varied. (a) defines the runtime as 50. (b) defines the runtime as 100. (c) defines the runtime as 500. (d) defines the runtime as 1000. (e) defines the runtime as 5000. (f) defines the runtime as 10000 (See also Reichhardt and Reichhardt 2015, Bechinger et al. 2016, and Reichhardt et al. 2018, under review).

Figure 20 shows how varying run time can affect the average velocity profile in the system as the run time is increased. With a drive force of 7.0 and at low run times, it is observed that the individual motion of the particles is quickly dampened out and the drive force almost exclusively contributes to the overall motion of the group. However, even at a steady state, there is a visible difference in the steady state behavior of Figure 20a and $20 \mathrm{~b}$ as the fluctuations in the average velocity are larger for the run time of 100 . As run time continues to increase, the average velocity 
profile begins to transition to a different phase as the drive force can no longer dampen out the run time as seen in Figure 20c and 20d. For very large run times, the average velocity becomes saturated with the run-and-tumble effect of the individual particles, promoting a continuously mixing orientation where the average velocity at the steady state of Figure 20e and 20f is 6.0, a value lower than defined drive force. Therefore, the characteristic effect of the run-and-tumble force is to model the individualistic and stochastic behavior a particle can exhibit, similar to how a human will change direction spontaneously in collective group given stimulus.

Now that these two competing effects are understood. We alter their parameters in our simulation to see how these forces contribute to the system and its structure. Given the rich variety of behaviors that occur in the simulation, we sought to characterize how these behaviors occur. The systems exhibit four basic behaviors: a jammed state, a completely phase separated state, a continuously mixing state and a laning state. 

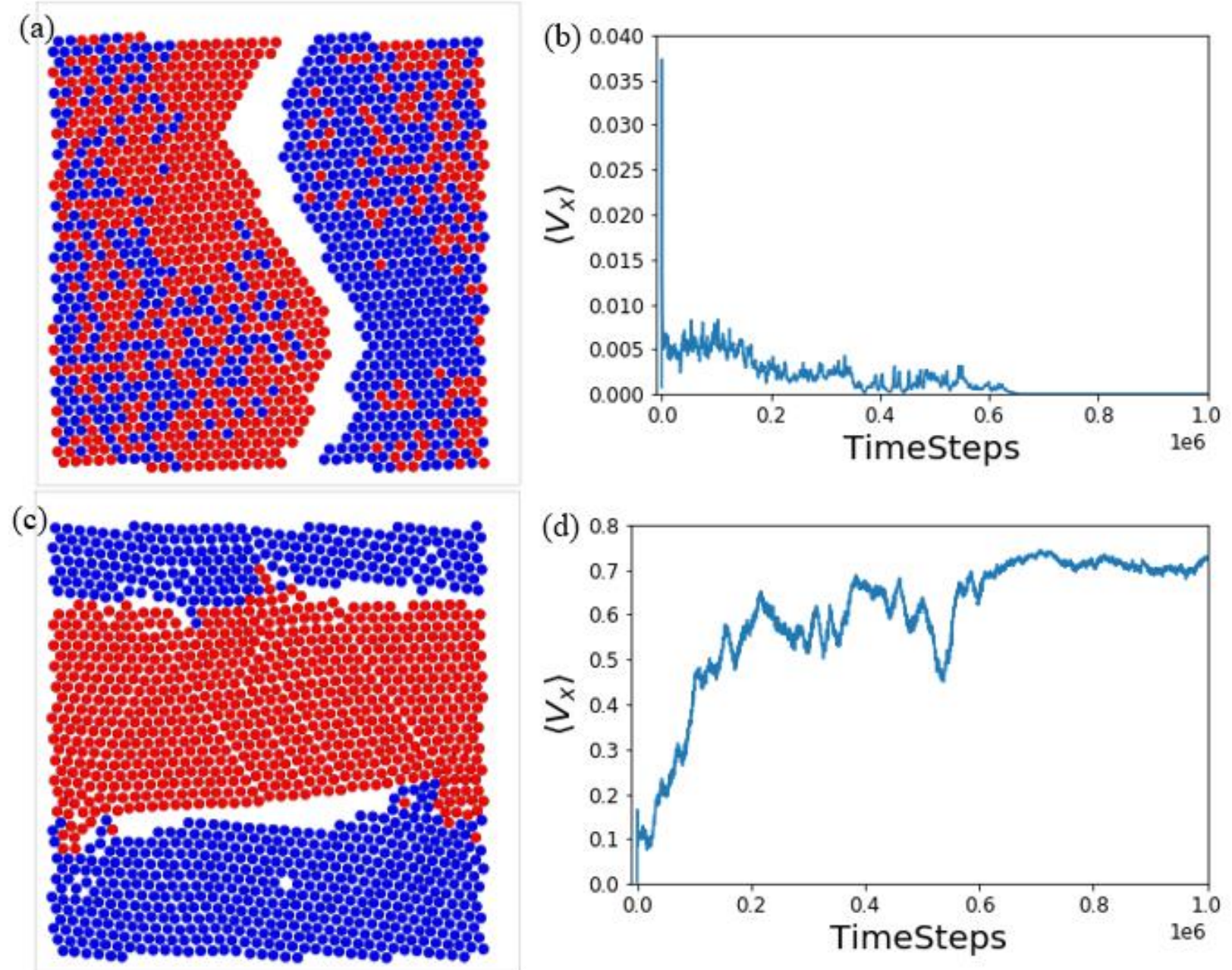

Figure 21: Possible Phases Observed in the System: (a) and (b) display the steady state phase of jamming particle configuration and the corresponding average velocity profile, respectively. $(c)$ and $(d)$ display the steady state phase separation of the particles and the corresponding average velocity profile, respectively (See also Reichhardt and Reichhardt 2016, Reichhardt and Reichhardt 2018, and Fig 12 in Reichhardt et al. 2018, under review). 

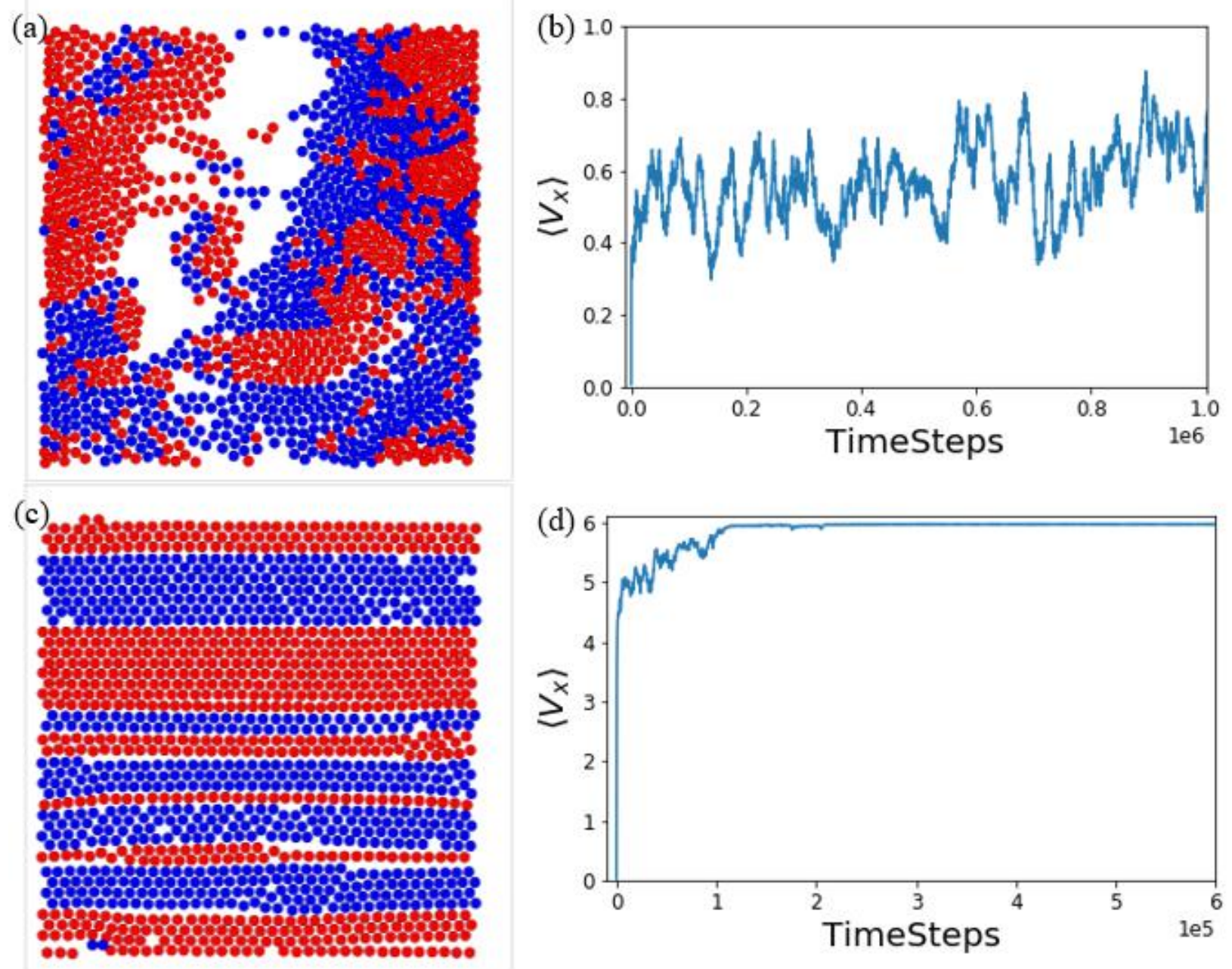

Figure 22: Possible Phases Observed in the System (continued): (a) and (b) display the steady state continuously mixing configuration of the system and the corresponding average velocity profile, respectively. (c) and (d) display the steady state laning orientation of the system and the corresponding average velocity profile, respectively (See also Reichhardt and Reichhardt 2016, Reichhardt and Reichhardt 2018, and Fig 12 in Reichhardt et al. 2018, under review).

Each of the behaviors has represented a relative crowd-based behavior that has been documented. Figure 21 and 22 outlines the various phases encountered when altering parameters in our model. With little to no drive force and quenched disorder, the disks become frozen in place and are considered pinned or jammed (Figure 21a). As observed in Figure 21b, the average profile begins with a sharp increase at the initiation of the simulation, but the velocity decreases quickly to a near zero value. At low drive forces, the disks form a large-scale phase separated state where particles organize into two groups where each group is comprised from one type of the particles (Figure 21c). As a result, the groups of particles move past each other with minimal resistance. The corresponding velocity plot shows how the particles mix at the beginning of the simulation 
but become self-organized as the average velocity converges on the defined drive force value (Figure 21d). As we continue to increase the drive force, the phase separation state transitions into a scattered, small-scale clustering state where the particles continuously mix in relatively even distribution as seen in Figure 22a. The average velocity profile displays stochastic fluctuations that are less that the defined drive force value (Figure 22b). The phase that occurs at high drive forces is referred to as laning (Figure 22c). The drive force quickly dampens out any contribution to individual disk movement and the particles begin to separate into well-defined lanes of the same type of particle. They pass by opposing particles relatively freely. This behavior is consistent with the average velocity profile as it can be observed that the fluctuations from particle interactions quickly dampen out as the average velocity converges to the drive force.

\section{iii. Evolution of the Phase Diagram with Particle Density}

Next, we consider the role of activity in a dense system. For the purpose of these simulations, we fixed the number of particles to be 1154 , approximately $70 \%$ of the system's available space. The average velocity and structure were measured. We know that the system displays four unique, self-organizing behaviors as the drive force and run-and-tumble force are varied. These must be considered as the system reaches steady state. Given that we collect the average velocity of one type of particle, we use this parameter to observe discrete changes in the steady state velocity average. 

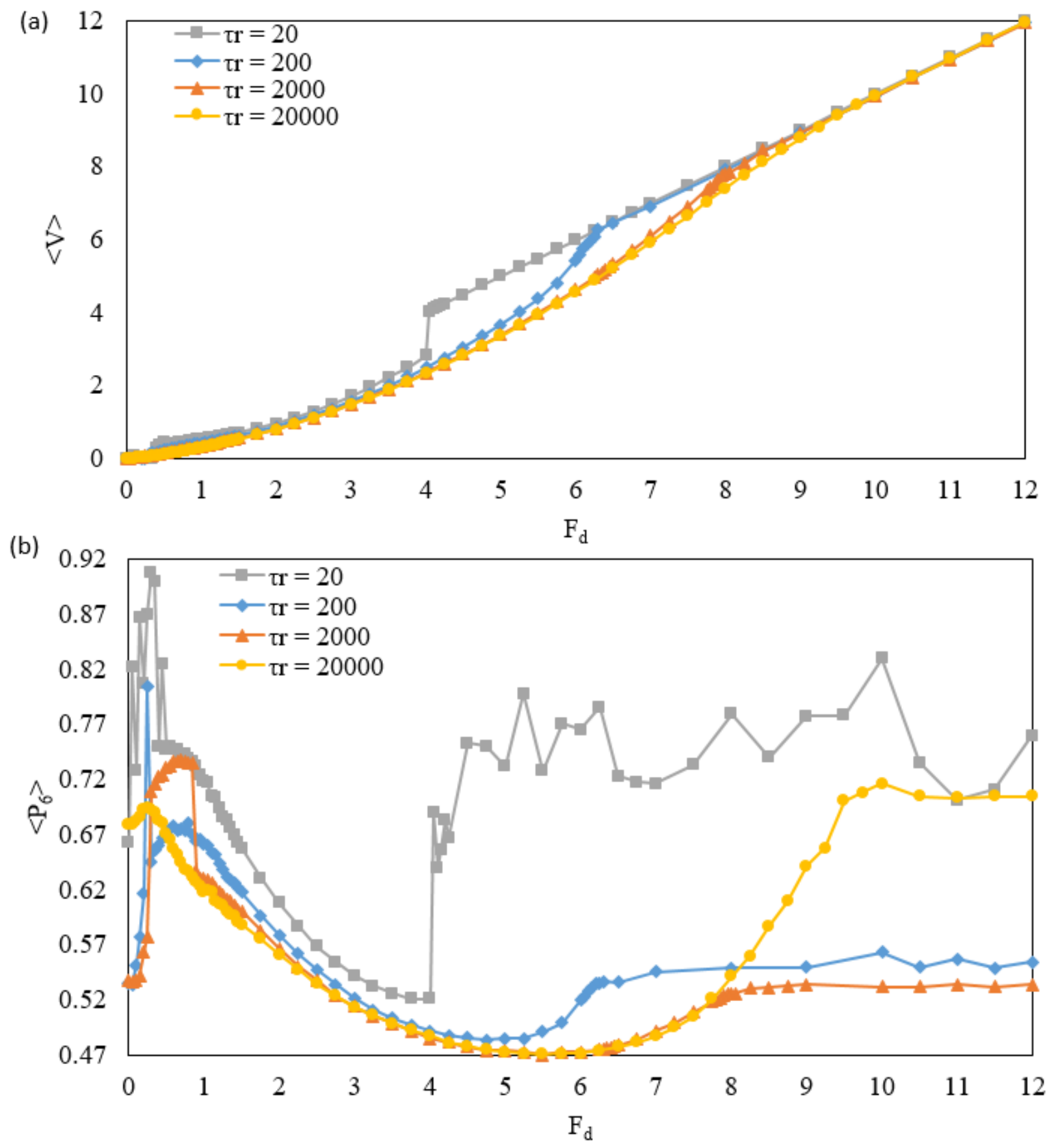

Figure 23: Average velocity and structure profiles of various run times: For one realization, a system of density $\varphi=70 \%$ with runtimes of 20,200, 2000, and 20000. (a) average velocity as a function of drive force and (b) structural measurement of the system at steady state as a function of drive force (See also Reichhardt and Reichhardt 2016, Reichhardt and Reichhardt 2018, and Fig 11 in Reichhardt et al. 2018, under review). Note that corresponding jumps in $P_{6}$ structure and average velocity indicate the transition of a phase behavior.

Figure 23a is the average velocity profile for the phase transition with respect to drive force. The phase transitions are a function of the run time and the drive force. This is trivial, given that the phases changes are a result of both parameters. This plot serves as a map to tune the parameters to 
specific structural behaviors. For laning behavior, the average velocity at the steady state obtains relatively the same value as the drive force. As the system enters the mixing phase, the average velocity value diverges from the drive force. As the system enters the phase separation phase, the average velocity converges back to the low drive force value. In the jammed state, the average velocity is zero. To prove that this transition can, also, be seen throughs structural measures. We use plot the fraction of sixfold coordinated particles, $\mathrm{P}_{6}$, as a function of drive force. This parameter is meant to measure the order of the structure of the system. Both plots display the same transition point in the simulations when the parameters are changed.

To complete the analysis, a phase diagram was developed to characterize the systems transitions through the various phase behaviors as a function of the run time and drive force.

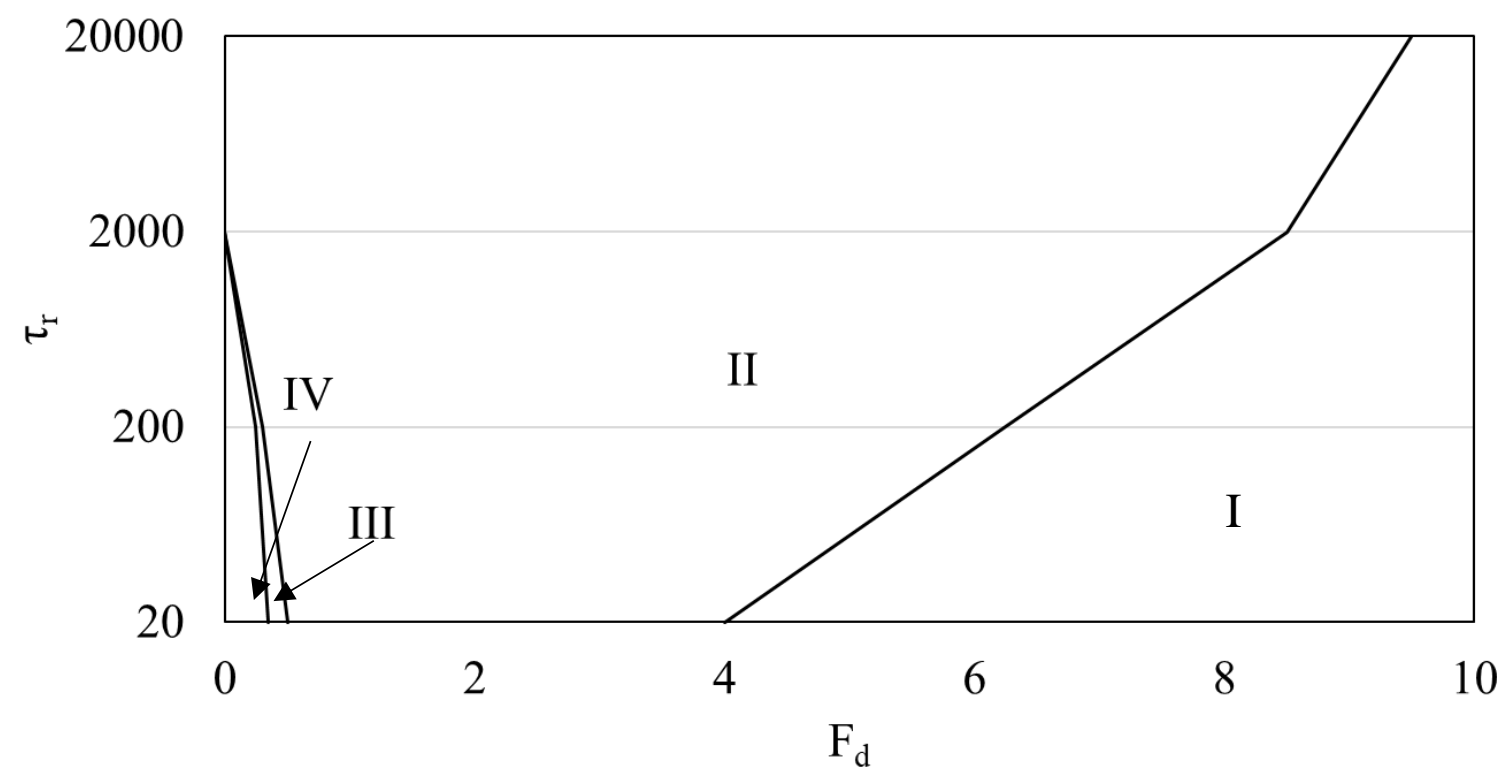

Figure 24: Phase Diagram of the Active Matter System: The phase diagram uses 1154 particles in the simulations, about $70 \%$ and is for one realization.. I denotes the region in which laning behavior occurs. II denote the region in which mixing behavior occurs. III denotes the region in which phase separation occurs. And IV denotes the region in which jamming occurs (See also Reichhardt and Reichhardt 2016, Reichhardt and Reichhardt 2018, and Fig 18 in Reichhardt et al. 2018, under review). Note that the peak in figure 21 for $\tau_{r}=20$ shows the phase change from IV (jammed state) at low $F_{d}$, to phase III (phase separated or large scale clustered) as $F_{d}$ is increased slightly. As drive force is continually increased, the activity of the individuals causes phase II (mixing) to occur. Finally, at large enough $F_{d}$ the systems enters an ordered, laning state where individual activity of the particles is mitigated by the overall global drive of the system. As $\tau_{r}$ is increased, the mixing state becomes more prevalent at a wider range of $F_{d}$ for the system density of $70 \%$.

Once the average velocity of the steady state values was plotted as a function of drive force, distinct changes in the value were noted as the transition points. This procedure was carried out across several runtimes. At the boundary of the transitions, the system took a much longer time to achieve a steady state behavior compared to simulation in the middle of the phase behavior. At large drive forces $(>9.5)$, the phase behavior self-organizes to a laning behavior across all run 
times. As the density of the system increases, the laning phase or self-organization of the particles begins to occur at higher drive force and becomes insensitive to system density at a drive force of about 9.5. As drive force is decreased, large values of run time begin to cause fluctuations in the steady state behavior where there are indications of a mixing behavior. The lowering of the drive force gives way to the increased activity of the particles, promoting a highly disordered state. Laning and mixing, typically, are the two dominating phases of the system. However, as we look at even lower drive forces, the jammed state and phase separation state are very sensitive to both parameters (Reichhardt et al 2018, under review). Further decreasing the drive force, the system becomes susceptible to large scale clustering which can induce a jammed state. Even though the particles still have their activity the system jams, expressing a freezing by heating mechanism. As we introduce more particles into the system, these jammed and phase separated region in the phase diagram grow. However, they become saturated at low drive force values $(<1.0)$ for the maximum possible system density. 


\section{Discussion: Disordered effects in active and inactive systems}

Active and inactive systems display several disordered effects with some similarities and differences. They share a quenched disordered state at the initiation of the simulations. We assign random characteristics in both examples. For our inactive system example, we introduce a quenched disordered parameter, critical strain energy release rate $G_{c}$ which can be referred to as the microstructural propensity to damage, in the fracture characteristics. In our active system example, the randomness is introduced in two ways. First, the particles are given a random starting orientation, giving a quenched disorder characteristic. Second, the run-and-tumble effect that dictates particle direction is randomized throughout the simulation.

Both systems evolve through the competition of the two opposing, controlled parameters. In the inactive system example, the competing parameters are the notch curvature and the disorder strength. In the limit of small disorder strength or very sharp notch curvature, disorder becomes unimportant. In the active system example, the competing parameters are the drive force imposed on the entire system and the individual run-and-tumble dynamics on the particles. In the limit of high-drive force or small runtime disorder is of an annealing nature and disappears at finite time in the system.

Active and inactive systems display very different disordered effects: Firstly, phase diagrams as parameters change are highly more complex in the active case. While in the inactive case, one observes two behaviors such as a brittle and a quasibrittle fracture. In active systems, one observes various phases such as laning, mixing, phase separation, and jamming. However, these are not the only phases that may be exhibited by the systems. There may be other phases present if further investigation of the systems continued. Another major difference is that a disordered response from the active system only requires an initial random condition to achieve a disordered state. However, for inactive systems, the initial condition of the actual mechanical response (stress, strain, etc.) is not affected as the load increases. In the next section, we underscore some important findings from the two disordered examples. 


\section{Conclusions}

These disordered systems and their models provide invaluable understanding in both material science and condensed matter research. The analysis of fracture in an inactive, disordered system with quenched characteristics leads to a phase diagram which could characterize the fracture behavior in alloys and superalloys using the competition of the notched geometry with the fluctuations in a materials fracture property. The purpose of these simulations is to understand and quantify how disorder effects crack formation and growth in intermetallics. Opposite to fracture

for an inactive system, an active matter system with quenched disorder was studied with intent to make comparison to large crowd behavior or swarming movement in an effort to characterize already seen behaviors in nature.

In the inactive system discussed, a simulation-based model of the idealized material is developed to obtain a better understanding of the realistic response that an alloyed material would experience in mode I fracture. The Weierstrass-Mandelbrot function was implemented in a material simulation software, DAMASK (Roters et al. 2012), towards the investigation of crack initiation in disordered microstructures that may deform elastically and plastically. A phase field model was implemented to control the critical strain energy release rate of each sample. Our modeling methods can carefully describe micromechanical material properties, in a constitutive manner, such as viscoplasticity, hardening, twinning, elastic anisotropy, and they may be used to simulate the fracture behavior. The fractal dimension of the stochastic, microstructural properties, $\mathrm{D}$, is increased from 2.85 to 2.995 . For brittle fracture, the theory of fracture mechanics expects that crack initiation would occur at the notch tip. But, this behavior is, also, exhibited at lower disorder strengths where the critical strain energy release rate variance is not large enough to have an effect on the initial crack formation location. At higher disorder strengths, the critical strain energy release rate variance is large enough to induce crack nucleation on the bulk sample where the stresses are higher than those present at the notch tip, classifying the fracture as quasibrittle. The relation between notch-driven and disorder-driven fracture is identified through the behavior during crack initiation and quantified from parameters like average stress, maximum stress, and damage evolution.

In the active matter system, a molecular dynamics model was used to obtain a better understanding of the large crowd behavior in a channel-type flow with bi-directional traffic. There are several characteristic equations that govern the behavior of the system including the particle to 
particle interaction force, approximating a hard surface interaction with a stiff spring force, the drive force, dictating the global flow direction uniformly applied to every particle, and the run and tumble force, giving the particles their active nature. From sweeping the parameter space, the observation that was consistent throughout all the simulations was that the particles, typically, followed the path of least resistance. When a single particle encountered a large cluster, it followed the boundaries of the cluster or permeated through the cluster's front when an opening presented itself. These behaviors can be largely attributed to similarities seen in large crowd sociology (Moussaid et al. 2009 and Helbing et al. 2000). We have shown that active matter system initialized with quench disorder develop ordered structures through various phase transitions. Starting at higher drives, the steady state phases form a laning behavior. As the drive force decreases, the structure of the particles transitions to mixed configuration. At even lower drive forces, the particles enter a phase separated state or jammed/ pinned state. In the future, disordered systems, active and inactive, may yield more ground-breaking understandings in today's realistic networks. 


\section{References}

[1] Alava, Mikko J., Phani KVV Nukala, and Stefano Zapperi. "Role of disorder in the size scaling of material strength." Physical review letters 100, no. 5 (2008): 055502.

[2] Amy. The Airport Phenomenon. August, 2010. Blog at Word Press.com. https://pronetowanderblog.com/2010/08/21/the-airport-phenomenon/

[3] Aranson, I. S., V. A. Kalatsky, and V. M. Vinokur. "Continuum field description of crack propagation." Physical review letters 85, no. 1 (2000): 118.

[4] Asaro, Robert J. "Crystal plasticity." Journal of applied mechanics 50, no. 4b (1983): 921.

[5] Asaro, Robert, and Vlado Lubarda. Mechanics of solids and materials. Cambridge University Press, 2006.

[6] Bazant, Z. P., and P. A. Pfeiffer. "Test of shear fracture and strain-softening in concrete." In Proceedings of the second symposium on interaction of non-nuclear munition with structures, Florida, USA, (1985): 254.

[7] Bazant, Zdenek P., and Jaime Planas. Fracture and size effect in concrete and other quasibrittle materials. Vol. 16. CRC press, 1997.

[8] Bechinger, C., Roberto Di Leonardo, Hartmut Löwen, Charles Reichhardt, Giorgio Volpe, and Giovanni Volpe. "Active particles in complex and crowded environments." Reviews of Modern Physics 88, no. 4 (2016): 045006.

[9] Bhattacharya, S., and Mark J. Higgins. "Dynamics of a disordered flux line lattice." Physical review letters 70, no. 17 (1993): 2617.

[10] Brotzu, A., F. Felli, and D. Pilone. "Effects of the manufacturing process on fracture behaviour of cast TiAl intermetallic alloys." Frattura ed Integrità Strutturale: Annals 2014: Fracture and Structural Integrity: Annals 20148 (2014).

[11] Bunde, Armin, and Shlomo Havlin, eds. Fractals and disordered systems. Springer Science \& Business Media, 2012.

[12] Campbell, J. P., R. O. Ritchie, and KT Venkateswara Rao. "The effect of microstructure on fracture toughness and fatigue crack growth behavior in $\gamma$-titanium aluminide based intermetallics." Metallurgical and Materials Transactions A 30, no. 3 (1999): 563.

[13] Carbone, Luigi, and P. Davide Cozzoli. "Colloidal heterostructured nanocrystals: Synthesis and growth mechanisms." Nano Today 5, no. 5 (2010): 449. 
[14] Carney, Luis R., and John J. Mecholsky. "Relationship between fracture toughness and fracture surface fractal dimension in AISI 4340 steel." Materials Sciences and Applications 4, no. 04 (2013): 258.

[15] Cates, M. E., and J. Tailleur. "When are active Brownian particles and run-and-tumble particles equivalent? Consequences for motility-induced phase separation." EPL (Europhysics Letters) 101, no. 2 (2013): 20010.

[16] Clemens, Helmut, and Svea Mayer. "Design, processing, microstructure, properties, and applications of advanced intermetallic TiAl alloys." Advanced Engineering Materials 15, no. 4 (2013): 191-215.

[17] Couzin, Iain D., and Nigel R. Franks. "Self-organized lane formation and optimized traffic flow in army ants." Proceedings of the Royal Society of London B: Biological Sciences 270, no. 1511 (2003): 139.

[18] Dimiduk, D. M. "Gamma titanium aluminide alloys — an assessment within the competition of aerospace structural materials." Materials Science and Engineering: A 263, no. 2 (1999): 281.

[19] Dzubiella, J., G. P. Hoffmann, and H. Löwen. "Lane formation in colloidal mixtures driven by an external field." Physical Review E 65, no. 2 (2002): 021402.

[20] El Kadiri, Haitham, M. F. Horstemeyer, J. B. Jordon, and Yibin Xue. "Fatigue crack growth mechanisms in high-pressure die-cast magnesium alloys." Metallurgical and Materials Transactions A 39, no. 1 (2008): 190.

[21] Escudero, Carlos, Christian A. Yates, Jerome Buhl, Iain D. Couzin, Radek Erban, Ioannis G. Kevrekidis, and Philip K. Maini. "Ergodic directional switching in mobile insect groups." Physical Review E 82, no. 1 (2010): 011926.

[22] Grote, D. L., S. W. Park, and M. Zhou. "Dynamic behavior of concrete at high strain rates and pressures: I. experimental characterization." International Journal of Impact Engineering 25, no. 9 (2001): 869.

[23] Han, Jianchao, Shulong Xiao, Jing Tian, Yuyong Chen, Lijuan Xu, Xiaopeng Wang, Yi Jia, Zhaoxin Du, and Shouzhen Cao. "Grain refinement by trace TiB2 addition in conventional cast TiAl-based alloy." Materials Characterization 106, (2015): 112.

[24] Helbing, Dirk, Illés J. Farkas, and Tamás Vicsek. "Freezing by heating in a driven mesoscopic system." Physical review letters 84, no. 6 (2000): 1240. 
[25] Herbold, Eric B., Jennifer L. Jordan, and N. N. Thadhani. "Effects of processing and powder size on microstructure and reactivity in arrested reactive milled Al+ Ni." Acta Materialia 59, no. 17 (2011): 6717.

[26] Hu, D. "Effect of composition on grain refinement in TiAl-based alloys." Intermetallics 9, no. 12 (2001): 1037.

[27] Kim, Young-Won, and Dennis M. Dimiduk. "Progress in the understanding of gamma titanium aluminides." JOM Journal of the Minerals, Metals and Materials Society 43, no. 8 (1991): 40.

[28] Kim, Young-Won. "Ordered intermetallic alloys, part III: gamma titanium aluminides." JOM Journal of the Minerals, Metals and Materials Society 46, no. 7 (1994): 30.

[29] Lapin, J. "TiAl-based alloys: Present status and future perspectives." In Conference proceedings METAL, vol. 19, no. 21.5, (2009).

[30] Lattuada, Marco, and T. Alan Hatton. "Synthesis, properties and applications of Janus nanoparticles." Nano Today 6, no. 3 (2011): 286.

[31] Lay, S., and A. Reza Yavari. "Evidence for disordered character of grain boundaries in a Ni3Al-based alloy during reordering." Acta materialia 44, no. 1 (1996): 35.

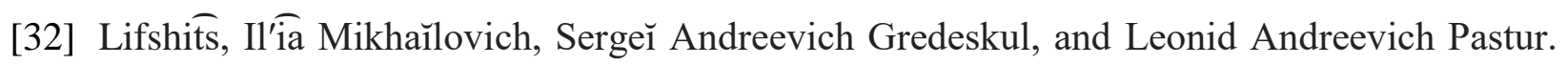
Introduction to the theory of disordered systems. Wiley-Interscience, 1988.

[33] Mandelbrot, B. B. "Fractals: form, chance and dimension." Fractals: form, chance and dimension., by Mandelbrot, BB. San Francisco (CA, USA): WH Freeman \& Co, 1979.

[34] Mandelbrot, Benoit B., Dann E. Passoja, and Alvin J. Paullay. "Fractal character of fracture surfaces of metals." Nature 308, no. 5961 (1984): 721.

[35] Marchetti, MC., A. Alan Middleton, Karl Saunders, and J. M. Schwarz. "Driven depinning of strongly disordered media and anisotropic mean-field limits." Physical review letters 91, no. 10 (2003): 107002.

[36] Moon, K., Richard T. Scalettar, and Gergely T. Zimányi. "Dynamical phases of driven vortex systems." Physical review letters 77, no. 13 (1996): 2778.

[37] Moulinec, H., and P. Suquet. "A numerical method for computing the overall response of nonlinear composites with complex microstructure." Computer methods in applied mechanics and engineering 157, no. 1-2 (1998): 69. 
[38] Moussaid, Mehdi, Simon Garnier, Guy Theraulaz, and Dirk Helbing. "Collective information processing and pattern formation in swarms, flocks, and crowds." Topics in Cognitive Science 1, no. 3 (2009): 469.

[39] Ochiai, S., S. Kuboshima, K. Morishita, H. Okuda, and T. Inoue. "Fracture toughness of Al 203 fibers with an artificial notch introduced by a focused-ion-beam." Journal of the European Ceramic Society 30, no. 7 (2010): 1659.

[40] Olson, C. J., C. Reichhardt, and Franco Nori. "Nonequilibrium dynamic phase diagram for vortex lattices." Physical review letters 81, no. 17 (1998): 3757.

[41] Pampillo, Carlos A. "Flow and fracture in amorphous alloys." Journal of Materials Science 10, no. 7 (1975): 1194.

[42] Papanikolaou, S., J. Thibault, C. Woodward, P. Shanthraj, and F. Roters. "Brittle to QuasiBrittle Transition and Crack Initiation Precursors in Disordered Crystals." arXiv preprint arXiv:1707.04332 (2017).

[43] Pardo, F., F. De La Cruz, P. L. Gammel, E. Bucher, and D. J. Bishop. "Observation of smectic and moving-Bragg-glass phases in flowing vortex lattices." Nature 396, no. 6709 (1998): 348.

[44] Reichhardt, Charles, and CJ Olson Reichhardt. "Active microrheology in active matter systems: Mobility, intermittency, and avalanches." Physical Review E 91, no. 3 (2015): 032313.

[45] Reichhardt, C., and CJ Olson Reichhardt. "Depinning and nonequilibrium dynamic phases of particle assemblies driven over random and ordered substrates: a review." Reports on Progress in Physics 80, no. 2 (2016): 026501.

[46] Reichhardt, C., and C. J. O. Reichhardt. "Velocity force curves, laning, and jamming for oppositely driven disk systems." Soft matter 14, no. 4 (2018): 490.

[47] Reichhardt, C., J. Thibault, S. Papanikolaou, and C. J. O. Reichhardt. "Laning and Clustering Transitions in Driven Binary Active Matter Systems." arXiv preprint arXiv:1805.06946 (2018).

[48] Ritchie, RO, and Peters JO. "Small fatigue cracks: mechanics, mechanisms and engineering applications." Materials Transactions 42, no. 1 (2001): 58.

[49] Roters, Franz, Philip Eisenlohr, Luc Hantcherli, Denny Dharmawan Tjahjanto, Thomas R. Bieler, and Dierk Raabe. "Overview of constitutive laws, kinematics, homogenization and 
multiscale methods in crystal plasticity finite-element modeling: Theory, experiments, applications." Acta Materialia 58, no. 4 (2010): 1152.

[50] Roters, F., P. Eisenlohr, C. Kords, D. D. Tjahjanto, M. Diehl, and D. Raabe. "DAMASK: the Düsseldorf Advanced MAterial Simulation Kit for studying crystal plasticity using an FE based or a spectral numerical solver." Procedia IUTAM 3 (2012): 3.

[51] Schlangen, E., and JGMi Van Mier. "Experimental and numerical analysis of micromechanisms of fracture of cement-based composites." Cement and concrete composites 14, no. 2 (1992): 105.

[52] Schlangen, H. E. J. G. "Experimental and numerical analysis of fracture processes in concrete." HERON 38, no. 2 (1993).

[53] Schmittmann, B., and R. K. P. Zia. "Driven diffusive systems. An introduction and recent developments." Physics reports 301, no. 1-3 (1998): 45.

[54] Shanthraj, Pratheek, Omid Rezvanian, and Mohammed A. Zikry. "Electrothermomechanical finite-element modeling of metal microcontacts in MEMS." Journal of Microelectromechanical Systems 20, no. 2 (2011): 371.

[55] Shanthraj, Pratheek, Luv Sharma, Bob Svendsen, Franz Roters, and Dierk Raabe. "A phase field model for damage in elasto-viscoplastic materials." Computer Methods in Applied Mechanics and Engineering 312 (2016): 167.

[56] Shekhawat, Ashivni, Stefano Zapperi, and James P. Sethna. "From damage percolation to crack nucleation through finite size criticality." Physical review letters 110, no. 18 (2013): 185505 .

[57] Stoloff, N. S., C. T. Liu, and S. C. Deevi. "Emerging applications of intermetallics." Intermetallics 8, no. 9-11 (2000): 1313.

[58] Tailleur, J., and M. E. Cates. "Statistical mechanics of interacting run-and-tumble bacteria." Physical review letters 100, no. 21 (2008): 218103.

[59] Tian, Yuan, Donald McAllister, Hendrik Colijn, Michael Mills, Dave Farson, Mark Nordin, and Sudarsanam Babu. "Rationalization of microstructure heterogeneity in Inconel 718 builds made by the direct laser additive manufacturing process." Metallurgical and Materials Transactions A 45, no. 10 (2014): 4470.

[60] Tseng, Ampere A. "Recent developments in micromilling using focused ion beam technology." Journal of Micromechanics and Microengineering 14, no. 4 (2004): R15. 
[61] Turnbull, David. "Under what conditions can a glass be formed?" Contemporary physics 10, no. 5 (1969): 473.

[62] Vajpai, Sanjay K., and Kei Ameyama. "A novel powder metallurgy processing approach to prepare fine-grained Ti-rich TiAl-based alloys from pre-alloyed powders." Intermetallics 42 (2013): 146.

[63] Walsh, P. F. "Fracture of plain concrete." Indian Concrete Journal 46, no. 11 (1972).

[64] Wang, Lei, Jun Shen, Yunpeng Zhang, and Hengzhi Fu. "Microstructure, fracture toughness and compressive property of as-cast and directionally solidified NiAl-based eutectic composite." Materials Science and Engineering: A 664 (2016): 188.

[65] Weierstrass, Karl. "Uber continuierliche Functionen eines reellen Argumentes, die für keinen Werth des lezteren einen bestimmten Differentialquotient besitzen." Math. Werke II (1895).

[66] Xie, Jun, Sugui Tian, Xiaoming Zhou, Jingjing Li, and Wuxiang Wang. "Microstructure and enduring fracture features of FGH95 Ni-base superalloy." Rare Metals 30 (2011): 447.

[67] Zehnder, A. T. "Fracture Mechanics (Lecture Notes in Applied and Computational Mechanics)." (2012). 